\title{
Bayesian Analysis of Inverted Kumaraswamy Mixture Model with Application to Burning Velocity of Chemicals
}

\author{
Farzana Noor (D), ${ }^{1}$ Saadia Masood (D), Mehwish Zaman (D), Maryam Siddiqa (D), \\ Raja Asif Wagan, ${ }^{3}$ Imran Ullah Khan ${ }^{(D)},{ }^{4}$ and Ahthasham Sajid ${ }^{5}{ }^{5}$ \\ ${ }^{1}$ Department of Mathematics \& Statistics, International Islamic University, Islamabad 45320, Pakistan \\ ${ }^{2}$ Department of Mathematics and Statistics, PMAS University of Arid Agriculture, Rawalpindi, Pakistan \\ ${ }^{3}$ Department of Information Technology, Faculty of ICT, \\ Baluchistan University of Information Technology Engineering and Management Sciences, Quetta, Pakistan \\ ${ }^{4}$ College of Underwater Acoustics Engineering, Harbin Engineering University, Harbin, Heilongjiang, China \\ ${ }^{5}$ Department of Computer Science, \\ Faculty of ICT Baluchistan University of Information Technology Engineering and Management Sciences, Quetta, Pakistan
}

Correspondence should be addressed to Imran Ullah Khan; khan@hrbeu.edu.cn

Received 29 January 2021; Revised 2 April 2021; Accepted 27 April 2021; Published 19 May 2021

Academic Editor: Dao B. Wang

Copyright (C) 2021 Farzana Noor et al. This is an open access article distributed under the Creative Commons Attribution License, which permits unrestricted use, distribution, and reproduction in any medium, provided the original work is properly cited.

Burning velocity of different chemicals is estimated using a model from mixed population considering inverted Kumaraswamy (IKum) distribution for component parts. Two estimation techniques maximum likelihood estimation (MLE) and Bayesian analysis are applied for estimation purposes. BEs of a mixture model are obtained using gamma, inverse beta prior, and uniform prior distribution with two loss functions. Hyperparameters are determined through the empirical Bayesian method. An extensive simulation study is also a part of the study which is used to foresee the characteristics of the presented model. Application of the IKum mixture model is presented through a real dataset. We observed from the results that Linex loss performed better than squared error loss as it resulted in lower risks. And similarly gamma prior is preferred over other priors.

\section{Introduction}

Mixture models appear as obvious candidates whenever datasets that consist of two or more heterogeneous populations are mixed together. Due to its modeling versatility, the finite mixture model has attracted a great deal of attention in the history of statistics. To analyze the heterogeneous nature of processes, the mixture models are comparatively more suitable than the simple models. A mixture model with finite components is suitable to use when data are overdispersed, to fit a zero-expansion model, to measure heavy-tailed density, and to test for heterogeneity in cluster analysis. Mixture models have been effectively used in many areas such as industrial engineering (Ali et al. [1]), biology (Bhattacharya [2]), social sciences (Harris [3]), economics (Jedidi et al. [4]), and reliability (Sultan et al. [5]). For more detail about the finite mixture models, see Everitt
[6], Ali [7], Feroze and Aslam [8], Zhang and Huang [9], Fundi et al. [10], Tripathi et al. [11], Noor et al. [12], and Feroze and Aslam [13].

Many researchers have provided valuable literature on inverted distribution, for example, Aljuaid [14] studied inverse Weibull, Noor and Aslam [15] analyzed inverse Weibull mixture distribution, Abd EL-Kader et al. [16] analyzed inverted Pareto type I distribution, Basheer [17] proposed generalized alpha power inverse Weibull distribution, and Hassan and Zaky [18] present study on estimation of entropy for inverse Weibull distribution under multiple censored data. Kumaraswamy [19] proposed a distribution which has widespread applications, particularly in situations that are bounded from below and above, such as individual's height, test scores, atmospheric temperature, and hydrological data. AL-Fattah et al. [20] obtained the IKum distribution from the Kumaraswamy distribution 
using the transformation $X=(1 / T)-1$ when random variable $T$ has Kumaraswamy distribution with $\alpha$ and $\beta$ as shape parameters. They discussed important properties of inverted Kumaraswamy distribution and obtained parameters of the proposed model by using MLE and Bayesian technique. The IKum distribution has a long tail to the right; as a result, it can effectively be used for long-term reliability predictions and producing optimistic predictions as compared to other distributions.

Censoring is an important factor of experiments measuring life/failure times. Censored samples are encountered in life test whenever the experimenter has some obligations on the cost or available time for the experiment. Different censoring schemes are used for different experiments, but type I censoring is the most commonly used censoring scheme.

Our aim is to analyze inverted Kumaraswamy distribution in a different way as no other work after AL-Fattah et al. [20] is found on the IKum distribution. We propose a mixture model whose component densities are formed by IKum density and estimate the parameters and reliability function of the mixture model under study using Bayesian as well as frequentist method.

\section{Methodology}

2.1. Two-Component Mixture Model of IKum Distribution. A random variable $X$ supposed to have a $k$ component mixture model is defined as follows:

$$
f(x)=\sum_{i=1}^{k} \delta_{i} f_{i}(X \mid \Theta),
$$

where $\Theta=\left(\delta_{i}, \alpha_{i}, \beta_{i}\right), i=1,2, \ldots, k$

The probability density function (pdf) and reliability function of the mixture model whose component densities are characterized by IKum distribution are given by

$$
\begin{array}{ll}
f(x ; \Theta)=\delta_{1} f_{1}\left(x ; \Theta_{1}\right)+\left(1-\delta_{1}\right) f_{2}\left(x ; \Theta_{2}\right), & 0<\delta_{1}<1, \\
R(x ; \Theta)=\delta_{1} R_{1}\left(x ; \Theta_{1}\right)+\left(1-\delta_{1}\right) R_{2}\left(x ; \Theta_{2}\right), & 0<\delta_{1}<1,
\end{array}
$$

where pdf and reliability function of $i^{\text {th }}$ IKum density, respectively, are

$$
\left\{\begin{array}{l}
f_{i}\left(x ; \Theta_{i}\right)=\alpha_{i} \beta_{i}\left(1+x_{i}\right)^{-\left(\alpha_{i}+1\right)}\left\{1-\left(1+x_{i}\right)^{-\alpha_{i}}\right\}^{\beta_{i}-1} \\
R_{i}\left(x ; \Theta_{i}\right)=\left[1-\left\{1-\left(1+x_{i}\right)^{-\alpha_{i} \beta_{i}}\right]\right. \\
x>0, \alpha_{i}, \beta_{i}>0, i=1,2 .
\end{array}\right.
$$

2.2. Sampling and Likelihood Function under Type 1 Censoring. Suppose $m$ items are taken from a population which is a mixture of two-component IKum model with prespecified termination time $T_{0}$. Let the test be conducted, $s$ items are failed from $m$ items, and $(m-s)$ items are still in working position. As per the work of Mendenhall and Hader [21], in many problems, only the futile (useless) items are easily marked as a family of first population and second population. For example, an engineer may divide failed items of electronic as a first population and second population on the basis of failure cause. From the whole population, $s_{1}$ units belong to the first population, $s_{2}$ are from the second population, and " $m-s$ " items do not give us any information about the population to which they belong to. It is obvious that $s=s_{1}+s_{2}$ are the number of uncensored items. Suppose that $x_{i j}$ denote the failure times of $j^{\text {th }}$ item which are belonging to the $i^{\text {th }}$ subpopulation and that $x_{i j} \leq t_{0}, i=1,2$ and $j=1, \ldots, s_{i}$.

The likelihood function for the IKum mixture model using the above-discussed sampling scheme given by Mendenhall and Hader [21] is given by

$$
\begin{aligned}
L(\Theta \mid x) \propto & \prod_{j=1}^{s_{1}} \delta_{1} f_{1}\left(x_{1 j} ; \Theta_{1}\right) \prod_{j=1}^{s_{2}}\left(1-\delta_{1}\right) f_{2}\left(x_{2 j} ; \Theta_{2}\right)\left\{\delta_{1} R_{1}\left(x ; \Theta_{1}\right)+\left(1-\delta_{1}\right) R_{2}\left(x ; \Theta_{2}\right)\right\}^{m-s} \\
= & \prod_{j=1}^{s_{1}} \delta_{1} \alpha_{1} \beta_{1}\left(1+x_{1 j}\right)^{-\left(\alpha_{1}+1\right)}\left\{1-\left(1+x_{1 j}\right)^{-\alpha_{1}}\right\}^{\beta_{1}-1} \prod_{j=1}^{s_{2}}\left(1-\delta_{1}\right) \alpha_{2} \beta_{2}\left(1+x_{2 j}\right)^{-\left(\alpha_{2}+1\right)} \\
& \left\{1-\left(1+x_{2 j}\right)^{-\alpha_{2}}\right\}^{\beta_{2}-1}\left[1-\delta_{1}\left\{1-\left(1+t_{o}\right)^{-\alpha_{1}}\right\}^{\beta_{1}}-\left(1-\delta_{1}\right)\left\{1-\left(1+t_{o}\right)^{-\alpha_{2}}\right\}^{\beta_{2}}\right]^{m-s},
\end{aligned}
$$

where $x=\left(\mathbf{x}_{11}, x_{12}, \ldots, x_{1 s_{1}} \mathbf{x}_{21}, x_{22}, \ldots, x_{2 s_{2}}\right)$ are uncensored observations for failure time.
2.3. Maximum Likelihood Estimation. Taking log of the likelihood function (4) and differentiating w.r.t. parameters 
result in five nonlinear equations. A solution of these nonlinear equations gives MLEs for the vector of parameters.
We use the SAS package to compute ML estimates of the parameter and their MSEs.

$$
\begin{aligned}
& \frac{\partial Q(\Theta \mid \mathbf{x})}{\partial \delta_{1}}=\frac{s_{1}}{\delta_{1}}-\frac{s_{2}}{\left(1-\delta_{1}\right)}-\frac{(m-s)\left(F_{2}(t)-F_{1}(t)\right)}{R(t)}, \\
& \frac{\partial Q(\Theta \mid \mathbf{x})}{\partial \alpha_{1}}=\frac{s_{1}}{\alpha_{1}}-\sum_{j=1}^{s_{1}} \ln \left(1+x_{1 j}\right)-\left(\beta_{1}-1\right) w_{1}(x)-\frac{(m-s) \delta_{1} \beta_{1} \phi_{1}(t) F_{1}(t)}{R(t)\left(1-(1+t)^{-\alpha_{1}}\right)}, \\
& \frac{\partial Q(\Theta \mid \mathbf{x})}{\partial \alpha_{2}}=\frac{s_{2}}{\alpha_{2}}-\sum_{j=1}^{s_{2}} \ln \left(1+x_{2 j}\right)-\left(\beta_{2}-1\right) w_{2}(x)-\frac{(m-s)\left(1-\delta_{1}\right) \beta_{2} \phi_{2}(t) F_{2}(t)}{R(t)\left(1-(1+t)^{-\alpha_{2}}\right)}, \\
& \frac{\partial Q(\Theta \mid \mathbf{x})}{\partial \beta_{1}}=\frac{s_{1}}{\beta_{1}}-\sum_{j=1}^{s_{1}} \ln \left(1-\left(1+x_{1 j}\right)^{-\alpha_{1}}\right)-\frac{(m-s) \delta_{1} F_{1}(t) \ln \left(1-(1+t)^{-\alpha_{1}}\right)}{R(t)}, \\
& \frac{\partial Q(\Theta \mid \mathbf{x})}{\partial \beta_{2}}=\frac{s_{2}}{\beta_{2}}-\sum_{j=1}^{s_{2}} \sum_{j=1}^{s_{2}} \ln \left(1-\left(1+x_{2 j}\right)^{-\alpha_{2}}\right)-\frac{(m-s)\left(1-\delta_{1}\right) F_{2}(t) \ln \left(1-(1+t)^{-\alpha_{2}}\right)}{R(t)},
\end{aligned}
$$

where

$$
\begin{aligned}
F_{i}(t) & =\left\{1-(1+t)^{-\alpha_{i}}\right\}^{\beta_{i}}, \\
w_{i}(x) & =\sum_{j=1}^{r_{i}} \frac{\left(1+x_{i j}\right)^{-\alpha_{i}} \ln \left(1+x_{i j}\right)}{\left(1-\left(1+x_{i j}\right)^{-\alpha_{i}}\right)}, \\
\phi_{i} & =(1+t)^{-\alpha_{i}} \ln (1+t), \\
R(t) & =\left[1-\delta_{1}\left\{1-\left(1+t_{o}\right)^{-\alpha_{1}}\right\}^{\beta_{1}}-\left(1-\delta_{1}\left\{1-\left(1+t_{o}\right)^{-\alpha_{2}}\right\}^{\beta_{2}}\right], \quad i=1,2 .\right.
\end{aligned}
$$

2.4. Bayes Estimation. The Bayesian approach is a powerful statistical tool used to reduce uncertainty in complex problems. Bayesian theory basically relies upon prior distribution and the use of loss functions. Loss function represents the loss incurred when the real parameter is derived from the estimated value. Square error loss function (SELF) used in the study is a symmetric loss function. In many situations, overestimation is more serious than underestimation, or vice versa. Asymmetric loss functions are those loss functions in which negative and positive errors of the same or different dimensions cause different losses. To compensate the situation, an asymmetric loss function is also used.

\subsubsection{Posterior Density Assuming Informative (Gamma)}

Prior. It is assumed that $\alpha_{i}$ and $\beta_{i}$ each have gamma prior distribution with $\left(a_{i}, b_{i}\right)$ and $\left(c_{i}, d_{i}\right)$ hyperparameters, respectively, and $\delta_{1}$ assumes a uniform prior so joint prior density for $\alpha_{1}, \alpha_{2}, \beta_{1}, \beta_{2}$, and $\delta_{1}$ is

$$
g(\Theta) \propto \alpha_{1}^{a_{1}-1} e^{-b_{1} \alpha_{1}} \alpha_{2}^{a_{2}-1} e^{-b_{2} \alpha_{2}} \beta_{1}^{c_{1}-1} e^{-d_{1} \beta_{1}} \beta_{2}^{c_{2}-1} e^{-d_{2} \beta_{2}}, \quad a_{i}, b_{i}, c_{i}, d_{i}>0, i=1,2 .
$$

Thus, posterior density using the likelihood function and joint prior in proportional form is as follows: 


$$
\begin{aligned}
p(\Theta \mid \mathbf{x}) \propto & \prod_{i=1}^{s 1} \delta_{1} \alpha_{1} \beta_{1}\left(1+x_{1 j}\right)^{-\left(\alpha_{1}+1\right)}\left\{1-\left(1+x_{1 j}\right)^{-\alpha_{1}}\right\}^{\beta_{1}-1} \prod_{i=1}^{s 2}\left(1-\delta_{1}\right) \alpha_{2} \beta_{2}\left(1+x_{2 j}\right)^{-\left(\alpha_{2}+1\right)}\left\{1-\left(1+x_{2 j}\right)^{-\alpha_{2}}\right\}^{\beta 2-1} \\
& {\left[1-\delta_{1}\left\{1-\left(1+t_{o}\right)^{-\alpha_{1}}\right\}^{\beta_{1}}-\left(1-\delta_{1}\right)\left\{1-\left(1+t_{o}\right)^{-\alpha_{2}}\right\}^{\beta_{2}}\right]^{m-s} \alpha_{1}^{a_{1}-1} e^{-b_{1} \alpha_{1}} \alpha_{2}^{a_{2}-1} e^{-b_{2} \alpha_{2}} \beta_{1}^{c_{1}-1} e^{-d_{1} \beta_{1}} \beta_{2}^{c_{2}-1} e^{-d_{2} \beta_{2}} } \\
& \alpha_{i}, \beta_{i}>0, a_{i}, b_{i}, c_{i}, d_{i}>0,0<\delta_{1}<1, i=1,2 .
\end{aligned}
$$

Integration of the posterior density does not produce estimators in compact and simple form; therefore, we use Lindley's approximation to obtain Bayes estimators, posterior risks, and reliability estimates for the shape parameters of the IKum mixture model.

2.4.2. Lindley's Procedure for Estimation of Parameters. Lindley [22] proposed an approximation known as Lindley's approximation used to conduct posterior analysis when posterior density involves a complex integral. In this approximation, Bayes estimator expands as a function that involves a posterior mode of $\widehat{\Theta}$. Lindley's approximation has been utilized by many authors for the estimation of the parameters for the simple as well as mixture models; see Jaheen [23], Ahmad et al. [24], Sultan et al. [25], etc.

Consider the following integral

$$
\int U(\Theta) e^{Q(\Theta)} \mathrm{d}(\Theta)
$$

where $\Theta=\left(\alpha_{1}, \alpha_{2}, \beta_{1}, \beta_{2}, \delta_{1}\right)$ is a vector of parameters, $U(\Theta)$ is an arbitrary function of $\Theta$, and $Q(\Theta)$ is the logarithm of a posterior function for $n$ observation. Lindley [22] suggested the following approximate Bayes estimator under SELF:

$$
\widetilde{U}_{\mathrm{BL}}(\Theta)=E[U(\Theta) \mid x] \approx U(\widehat{\Theta})+\frac{1}{2} \sum_{i=1}^{m} \sum_{j=1}^{m}\left\{U_{i j}(\widehat{\Theta}) \tau_{i j}+\frac{1}{2} \sum_{i=1}^{m} \sum_{j=1}^{m} \sum_{s=1}^{m} \sum_{l=1}^{m} Q_{i j s}(\widehat{\Theta}) U_{l}(\widehat{\Theta}) \tau_{i j} \tau_{s l}\right\},
$$

where $i, j, s=1,2, \ldots, m$

$$
\begin{aligned}
U_{i}(\widehat{\Theta}) & =\left.\frac{\partial U(\Theta)}{\partial \theta_{i}}\right|_{\Theta=\widehat{\theta}}, \\
U_{i j}(\widehat{\Theta}) & =\left.\frac{\partial^{2} U(\Theta)}{\partial \theta_{i} \partial \theta_{j}}\right|_{\Theta=\hat{\theta}}, \\
\tau_{i j} & =(-Q)_{m * m}^{-1}=\sum_{m * m}, \\
Q_{i j}(\widehat{\Theta}) & =\left.\frac{\partial^{2} Q(\Theta)}{\partial \theta_{i} \partial \theta_{j}}\right|_{\Theta=\widehat{\theta}}, \\
Q_{i j s}(\widehat{\Theta}) & =\left.\frac{\partial^{3} Q(\Theta)}{\partial \theta_{i} \partial \theta_{j} \partial \theta_{s}}\right|_{\Theta=\hat{\theta}} .
\end{aligned}
$$

All the functions on the right-hand side are to be obtained as the posterior mode. $Q_{i j s}$ is given in Appendix B. Parameters of the proposed IKum mixture model using Lindley's approximation may be obtained as

$$
\tilde{U}(\Theta)=U(\widehat{\Theta})+\frac{1}{2}\left(D+\sum_{i=1}^{5} B_{i} A_{k}\right), \quad i=1,2, \ldots, 5,
$$

$$
\begin{aligned}
D & =\sum_{j=1}^{5} \sum_{k=1}^{5} U_{i}(\Theta) \tau_{i k}, \\
A_{k} & =\sum_{i=1}^{5} U_{i}(\Theta) \tau_{i k}, \\
B_{i} & =\sum_{k=1}^{5} \tau_{k k} Q_{k k i}(\Theta)+2\left\{\sum_{k=2}^{5} \tau_{i k} Q_{i k i}(\Theta)+E_{i}\right\} \\
E_{i} & =\tau_{23} Q_{23 i}+\tau_{24} Q_{24 i}+\tau_{25} Q_{25 i}+\tau_{34} Q_{34 i}+\tau_{35} Q_{35 i}+\tau_{45} Q_{45 i} .
\end{aligned}
$$

After equating $U(\Theta), U_{i k}=0, i, k=1,2, \ldots, 5$, and $D=0$, so the Bayes estimators of parameters $\alpha_{1}, \alpha_{2}, \beta_{1}, \beta_{2}$, and $\delta_{1}$ of the IKum mixture model under SELF are given by

$$
\begin{aligned}
& \widetilde{\delta}_{1(\mathrm{SELF})}=\widehat{\delta}_{1}+\frac{1}{2} \sum_{i=1}^{5} B_{i} \tau_{i 1}, \\
& \widetilde{\alpha}_{1(\mathrm{SELF})}=\widehat{\alpha}_{1}+\frac{1}{2} \sum_{i=1}^{5} B_{i} \tau_{i 2}, \\
& \widetilde{\alpha}_{2(\mathrm{SELF})}=\widehat{\alpha}_{2}+\frac{1}{2} \sum_{i=1}^{5} B_{i} \tau_{i 3}, \\
& \widetilde{\beta}_{1(\mathrm{SELF})}=\widehat{\beta}_{1}+\frac{1}{2} \sum_{i=1}^{5} B_{i} \tau_{i 4},
\end{aligned}
$$

where 


$$
\tilde{\beta}_{2(\text { SELF) }}=\widehat{\beta}_{2}+\frac{1}{2} \sum_{i=1}^{5} B_{i} \tau_{i 5}
$$

where $B_{i}, i=1,2, \ldots, 5$ are given earlier and $\tau_{i k}$ are the elements of the inverse of the matrix $Q_{i k}$. Posterior risk under SELF is the variance which can be evaluated as $E[U(\Theta) \mid x]^{2}-(E[U(\Theta) \mid x])^{2}$ by using Mathematica 12.
Under Linex loss function, equation (14) can be written as

$$
E_{\Theta}\left(e^{-\eta \Theta}\right)=\int e^{(-\eta \Theta)} U(\Theta) e^{Q(\Theta)} \mathrm{d}(\Theta) .
$$

The Bayes estimators of IKum distribution under LLF are

$$
\begin{gathered}
\widetilde{U}_{\mathrm{LLF}}(\Theta)=-\frac{1}{\eta} \ln \left\{e^{(-\eta \Theta)}+\frac{1}{2}\left(D_{\mathrm{LLF}}+\sum_{i=1}^{5} B_{i} A_{i}\right)\right\}, \\
\tilde{\delta}_{1(\mathrm{LLF})}=-\frac{1}{\eta} \ln \left\{e^{-\eta p_{1}}+\frac{1}{2}\left(U_{11}+\sum_{i=1}^{5} B_{i} \tau_{i 1}\right)\right\}, \\
\widetilde{\alpha}_{1(\mathrm{LLF})}=-\frac{1}{\eta} \ln \left\{e^{-\eta \alpha_{1}}+\frac{1}{2}\left(U_{22}+\sum_{i=1}^{5} B_{i} \tau_{i 2}\right)\right\}, \\
\tilde{\alpha}_{2(\mathrm{LLF})}=-\frac{1}{\eta} \ln \left\{e^{-\eta \alpha_{2}}+\frac{1}{2}\left(U_{33}+\sum_{i=1}^{5} B_{i} \tau_{i 3}\right)\right\}, \\
\tilde{\beta}_{1(\mathrm{LLF})}=-\frac{1}{\eta} \ln \left\{e^{-\eta \beta_{1}}+\frac{1}{2}\left(U_{44}+\frac{1}{2} \sum_{i=1}^{5} B_{i} \tau_{i 4}\right)\right\}, \\
\tilde{\beta}_{2(\mathrm{LLF})}=-\frac{1}{\eta} \ln \left\{e^{-\eta \beta_{1}}+\frac{1}{2}\left(U_{55}+\frac{1}{2} \sum_{i=1}^{5} B_{i} \tau_{i 5}\right)\right\},
\end{gathered}
$$

and posterior risks under LLF are

$$
\begin{aligned}
& \rho\left(\widehat{\delta}_{1(\mathrm{LLF})}\right)=\ln \left\{e^{\left(-\eta \delta_{1}\right)}+\frac{1}{2}\left[D_{\mathrm{LLF}}+\sum_{i=1}^{5} B_{i} A_{i}\right]\right\}+\eta\left(\delta_{1(\mathrm{MLE})}+\frac{1}{2} \sum_{i=1}^{5} B_{i} \tau_{i 1}\right), \\
& \rho\left(\widehat{\alpha}_{1(\mathrm{LLF})}\right)=\ln \left\{e^{\left(-\eta \alpha_{1}\right)}+\frac{1}{2}\left(D_{\mathrm{LLF}}+\sum_{i=1}^{5} B_{i} A_{i}\right)\right\}+\eta\left(\alpha_{1(\mathrm{MLE})}+\frac{1}{2} \sum_{i=1}^{5} B_{i} \tau_{i 2}\right), \\
& \rho\left(\widehat{\alpha}_{2(\mathrm{LLF})}\right)=\ln \left\{e^{\left(-\eta \alpha_{2}\right)}+\frac{1}{2}\left[D_{\mathrm{LLF}}+\sum_{i=1}^{5} B_{i} A_{i}\right]\right\}+\eta\left(\alpha_{2(\mathrm{MLE})}+\frac{1}{2} \sum_{i=1}^{5} B_{i} \tau_{i 3}\right), \\
& \rho\left(\widehat{\beta}_{1(\mathrm{LLF})}\right)=\ln \left\{e^{\left(-\eta \beta_{1}\right)}+\frac{1}{2}\left[D_{\mathrm{LLF}}+\sum_{i=1}^{5} B_{i} A_{i}\right]\right\}+\eta\left(\beta_{1(\mathrm{MLE})}+\frac{1}{2} \sum_{i=1}^{5} B_{i} \tau_{i 4}\right), \\
& \rho\left(\widehat{\beta}_{2(\mathrm{LLF})}\right)=\ln \left\{e^{\left(-\eta \beta_{2}\right)}+\frac{1}{2}\left[D_{\mathrm{LLF}}+\sum_{i=1}^{5} B_{i} A_{i}\right]\right\}+\eta\left(\beta_{2(\mathrm{MLE})}+\frac{1}{2} \sum_{i=1}^{5} B_{i} \tau_{i 5}\right) .
\end{aligned}
$$


$B_{i}$ are defined earlier, $\tau_{i k}$ are the elements of the inverse of the matrix $Q_{i k}$, and $D_{\mathrm{LLF}}$ and $l_{i}, k_{i}, m_{i}, z_{i}, \theta_{i}$ where $i=1,2$ are given in Appendix B.

2.4.3. Posterior Density Assuming Informative (Inverse Beta) Prior. It is assumed that the shape parameters $\alpha_{i}$ and $\beta_{i}$ have inverse beta prior with hyperparameters $a_{i}, b_{i}$, and $c_{i} d_{i}$, respectively,

$$
\begin{aligned}
& g\left(\alpha_{i}\right) \propto \alpha_{i}^{\left(a_{i}-1\right)}\left(1-\alpha_{i}\right)^{-\left(a_{i}+b_{i}\right)} \alpha_{i}>0, \quad i=1,2, \\
& g\left(\beta_{i}\right) \propto \beta_{i}^{\left(c_{i}-1\right)}\left(1-\beta_{i}\right)^{-\left(c_{i}+\alpha_{i}\right)} \beta_{i}>0, \quad i=1,2 .
\end{aligned}
$$

Joint prior density for $\alpha_{i}, \beta_{i}$, and $\delta_{i}$, is given by

$$
g(\Theta) \propto \alpha_{i}^{\left(a_{i}-1\right)}\left(1-\alpha_{i}\right)^{-\left(a_{i}+b_{i}\right)} \beta_{i}^{\left(c_{i}-1\right)}\left(1-\beta_{i}\right)^{-\left(c_{i}+d_{i}\right)} .
$$

Combining the likelihood function (4) and joint prior (32), the following joint posterior density of the IKum mixture model is obtained:

$$
\begin{aligned}
& p(\Theta \mid x) \propto \sum_{l=0}^{m-s} \sum_{n=0}^{l}\left(\begin{array}{c}
m-s \\
l
\end{array}\right)\left(\begin{array}{c}
l \\
n
\end{array}\right)(-1)^{l} \delta_{1}^{s 1+l-n}\left(1-\delta_{1}\right)^{s 2+n} \alpha_{1}^{\left(a_{1}-1\right)}\left(1-\alpha_{1}\right)^{-\left(a_{1}+b_{1}\right)} \beta_{1}^{\left(c_{1}-1\right)}\left(1-\beta_{1}\right)^{-\left(c_{1}+d_{1}\right)} \alpha_{2}^{\left(a_{2}-1\right)} \\
&\left(1-\alpha_{2}\right)^{-\left(a_{2}+b_{2}\right)} \beta_{2}^{\left(c_{2}-1\right)}\left(1-\beta_{2}\right)^{-\left(c_{2}+d_{2}\right)} \operatorname{Exp}\left[-\left\{2 \alpha_{1} \sum_{j=1}^{s_{1}} \ln \left(1+x_{1 j}\right)+\sum_{j=1}^{s_{1}} \ln \left(1-\left(1+x_{1 j}\right)^{-\alpha_{1}}\right)\right\}\right] \\
& \operatorname{Exp}\left[-\left\{2 \alpha_{2} \sum_{j=1}^{s_{2}} \ln \left(1+x_{2 j}\right)+\sum_{j=1}^{s_{2}} \ln \left(1-\left(1+x_{2 j}\right)^{-\alpha_{2}}\right)\right\}\right] \operatorname{Exp}\left[\beta_{1}\left\{\sum_{j=1}^{s} \ln \left(1-\left(1+x_{1 j}\right)^{-\alpha_{1}}\right)+(j-n) \ln \left(1-(1+t)^{-\alpha_{1}}\right)\right\}\right] \\
& \operatorname{Exp}\left[\beta_{2}\left\{\sum_{j=1}^{s 2} \ln \left(1-\left(1+x_{2 j}\right)^{-\alpha_{2}}\right)+n \ln \left(1-(1+t)^{-\alpha_{2}}\right)\right\}\right], \quad \alpha_{1}>0, \alpha_{2}>0, \beta_{1}>0, \beta_{2}>0,0<\delta_{1}<1 .
\end{aligned}
$$

The posterior density in equation (33) again does not produce Bayes estimators in explicit form, so we use Lindley's approximation given in equation (14). The final form of Bayes estimators, posterior risks, and reliability function of IKum distribution under SELF and LLF assuming informative (inverse beta) prior is the same as given in (14)-(30).

2.5. Posterior Density Assuming Noninformative (Uniform) Prior. Noninformation priors are an important part of the
Bayesian tool and are considered for Bayesian analysis when there is little or no prior information available.

Let $\alpha_{i}, \beta_{i} \sim U(0, \infty)$, and $\delta_{i} \sim U(0,1), i=1,2$

So the joint prior is

$$
g(\Theta) \propto 1 .
$$

Assuming independence combining the prior with likelihood function (4). The joint posterior density of $\alpha_{i}, \beta_{i}$, and $\delta_{i}$ is obtained as

$$
\begin{aligned}
p(\Theta \mid \mathbf{x}) \propto & \prod_{i=1}^{s 1} \delta_{1} \alpha_{1} \beta_{1}\left(1+x_{1 j}\right)^{-\left(\alpha_{1}+1\right)}\left\{1-\left(1+x_{1 j}\right)^{-\alpha_{1}}\right\}^{\beta_{1}-1} \prod_{i=1}^{s 2}\left(1-\delta_{1}\right) \alpha_{2} \beta_{1}\left(1+x_{2 j}\right)^{-\left(\alpha_{2}+1\right)}\left\{1-\left(1+x_{2 j}\right)^{-\alpha_{2}}\right\}^{\beta 2-1} \\
& {\left[1-\delta_{1}\left\{1-\left(1+t_{o}\right)^{-\alpha_{1}}\right\}^{\beta_{1}}-\left(1-\delta_{1}\right)\left\{1-\left(1+t_{o}\right)^{-\alpha_{2}}\right\}^{\beta_{2}}\right]^{m-s}, \quad \alpha_{1}, \alpha_{2}, \beta_{1}, \beta_{2}>0,0<\delta_{1}<1 . }
\end{aligned}
$$

Mathematical expressions of the Bayes estimators and posterior risk can be obtained by assigning hyperparameters a zero value in the expressions of Bayes estimators and posterior risks in Sections 2.4.1 and 2.4.3 under SELF and LLF.

2.6. Reliability Estimation. The objective of assessing the reliability of estimates is to determine how much of the variability in the data is due to errors in measurement. And how much is in the true parameters. Approximate Bayes estimator of reliability function of IKum at some value $t$ can be obtained as

$$
\widehat{R}(t)=R(x ; \Theta)+\frac{1}{2}\left(D_{R}+\sum_{i=1}^{5} B_{i} A_{i}\right) .
$$


Here,

$$
\begin{aligned}
D_{R}= & \left\{l_{1}\left(\tau_{12}+\tau_{21}\right)+l_{2}\left(\tau_{13}+\tau_{31}\right)+k_{1}\left(\tau_{14}+\tau_{41}\right)+k_{2}\left(\tau_{15}+\tau_{51}\right)+\delta_{1} m_{1}\left(\tau_{24}+\tau_{42}\right)+\right. \\
& \left.\left(1-\delta_{1}\right) m_{2}\left(\tau_{35}+\tau_{53}\right)+\delta_{1} \theta_{1} \tau_{22}+\left(1-\delta_{1}\right) \theta_{2} \tau_{33}+\delta_{1} z_{1} \tau_{44}+\left(1-\delta_{1}\right) z_{2} \tau_{55}\right\}, \\
A_{i}= & {\left[\left\{F_{2}(t)-F_{1}(t)\right\} \tau_{i 1}+\delta_{1} l_{1} \tau_{i 2}+\left(1-\delta_{1}\right) l_{2} \tau_{i 3}+\delta_{1} k_{1} \tau_{i 4}+\left(1-\delta_{1}\right) k_{2} \tau_{i 5}\right], \quad i=1,2,3,4,5, }
\end{aligned}
$$

where $B_{i}$ is defined earlier, $\tau_{i k}$ are the elements of the invers of the matrix $Q_{i k}$, and $l_{i}, k_{i}, m_{i}, z_{i}, \theta_{i}(i=1,2)$ are given in Appendix B.

\section{Results and Discussion}

3.1. Monte Carlo Simulation. Simulation is performed to get insight into properties/trends of the obtained Bayes estimators. For this purpose, a Monte Carlo simulation is performed for 1000 samples of size $n=30,50$, and 100 for each selection of the vector of parameters $\Theta=\left(\delta_{1}, \alpha_{1}, \alpha_{2}\right.$, $\left.\beta_{1}, \beta_{2}\right)(0.4,1,1.5,0.8,0.5),(0.4,2,3,1.3,1.5)$ using the inverse transformation method as follows:

Generate a random sample of different selected sample sizes from the proposed mixture model using the inverse transformation method

If $u_{1}<\delta_{1}$, then use $u_{1}$ to generate random variate $x$ from the mixture of two-component IKum as $x=\left(\left(1-u_{1}^{1 / \beta_{1}}\right)^{-\left(1 / \alpha_{1}\right)}-1\right)$.

If $u_{1} \geq \delta_{1}$, then use $u_{2}$ to generate random variate $x$ from the mixture of two-component IKum as $x=\left(\left(1-u_{2}^{1 / \beta_{2}}\right)^{-\left(1 / \alpha_{2}\right)}-1\right)$.

Select a sample censored at a fixed test termination time $t$ and only take censored observations.

For the different choice of parameters, hyperparameter for the informative priors (gamma) are selected for $i=1,2$ to satisfy $E\left(\beta_{i}\right)=c_{i} / d_{i}$ and inverse beta $E\left(\beta_{i}\right)=c_{i} /\left(d_{i}-1\right)$,

$$
\begin{aligned}
& E\left(\beta_{1}\right)=\frac{c_{1}}{d_{1}}=\frac{5}{2}, \\
& E\left(\beta_{2}\right)=\frac{c_{2}}{d_{2}}=2, \\
& E\left(\beta_{1}\right)=\frac{c_{1}}{d_{1}}=\frac{10}{2}, \\
& E\left(\beta_{2}\right)=\frac{c_{2}}{d_{2}}=\frac{6}{2} .
\end{aligned}
$$

The above steps are repeated 1000 times. The Bayes estimates are computed over 1000 repetitions by averaging the estimate and the squared deviation, respectively. Estimates are computed using two informative (gamma and inverse beta) priors and uniform noninformative prior.

Results presented in Tables 1 and 2 (Appendix A) are obtained through simulation procedure which narrates the properties of the derived Bayes estimators and posterior risks of parameters and reliability estimate of the IKum mixture model. Different sample sizes, i.e., $n=30,50$, and 100 are taken to perform a simulation study. It is observed that as we increase the sample size, the estimate of parameters converges to a true parametric value. It is also observed that the use of LLF assuming gamma prior produces less posterior risk, hence can be thought of as a best loss function. An experimenter always tries to choose such a loss function for which he has to bear the minimum loss for estimation. In the same context, gamma prior resulted in smaller posterior risks as compared to other priors.

Bayes estimates are found overestimated for few cases and underestimated for few cases. It is all because the complex mixture model of IKum densities is considered and these Bayes estimates are obtained by using the approximate method. MLEs obtained in the simulation study are found to be somewhat inconsistent, and when compared with Bayes estimates, we found that PRs are lesser than MSEs of MLEs.

Results of simulation are also exhibited graphically which are given in Figure 1. From graphs of simulated Bayes estimates, we can see that we almost get the same results as obtained under simulation study numerically. It means we can obtain the same Bayes estimates through graphs.

3.2. Real Dataset Example. This dataset consists of $56 \mathrm{ob}-$ servations related to the burning velocity of different chemical materials. The burning speed/velocity is the laminar flame speed under the specified composition, temperature, and pressure conditions. It decreases as the inhibitor concentration increases and can be checked by analyzing the pressure distribution in the spherical vessel and by observing the flame propagation directly. Data related to burning velocity $(\mathrm{cm} / \mathrm{sec})$ of different chemical materials are given in Table 3 and are available at http:// www.cheresources.com/mists.pdf.

The following information is extracted from the above real data to analyze the mixture of the IKum model when $T=85$ : 
TABLE 1: BEs, PRs, and reliability estimates using UP, IBeta, and gamma prior under LLF and SELF for parameters $\left(\delta_{1}, \alpha_{1}, \alpha_{2}, \beta_{1}, \beta_{2}\right)(0.4$, $1.5,1,0.8,0.5)$ and $T=5$.

\begin{tabular}{|c|c|c|c|c|c|c|c|c|}
\hline \multirow{3}{*}{$n$} & \multirow{3}{*}{ Parameters } & \multirow{3}{*}{ MLEs } & \multicolumn{6}{|c|}{ Bayes estimates } \\
\hline & & & & SELF & & & LLF & \\
\hline & & & UP & IBeta & Gamma & UP & IBeta & Gamma \\
\hline \multirow{12}{*}{30} & \multirow{2}{*}{$\widehat{\alpha}_{1}$} & 1.38324 & 1.33580 & 1.29420 & 1.28110 & 1.35901 & 1.30142 & 1.29010 \\
\hline & & 0.18960 & 0.02753 & 0.04292 & 0.04842 & 0.02675 & 0.04129 & 0.04720 \\
\hline & \multirow{2}{*}{$\widehat{\alpha}_{2}$} & 2.91612 & 0.95686 & 0.95498 & 0.95418 & 0.96086 & 0.95680 & 0.95581 \\
\hline & & 0.60725 & 0.00188 & 0.00204 & 0.00212 & 0.00188 & 0.00187 & 0.00201 \\
\hline & \multirow{2}{*}{$\widehat{\beta}_{1}$} & 0.74762 & 0.65793 & 0.68732 & 0.69473 & 0.65693 & 0.68702 & 0.69703 \\
\hline & & 0.04374 & 0.004022 & 0.004345 & 0.00451 & 0.00401 & 0.00424 & 0.00401 \\
\hline & \multirow{2}{*}{$\widehat{\beta}_{2}$} & 0.49495 & 0.43708 & 0.43455 & 0.43332 & 0.43707 & 0.43454 & 0.43331 \\
\hline & & 0.01443 & 0.00402 & 0.00434 & 0.00451 & 0.00402 & 0.00434 & 0.00450 \\
\hline & \multirow{2}{*}{$\widehat{\delta}_{1}$} & 0.429810 & 0.42820 & 0.428557 & 0.42874 & 0.41087 & 0.41087 & 0.41087 \\
\hline & & 0.00832 & 0.00084 & 0.00012 & 0.00087 & 0.00012 & 0.00012 & 0.00012 \\
\hline & \multirow{2}{*}{$\widehat{R}(t)$} & 0.029982 & 0.037247 & 0.037346 & 0.037267 & 0.037312 & 0.037283 & 0.037266 \\
\hline & & 0.001955 & 0.001369 & 0.001361 & 0.001367 & 0.001364 & 0.001366 & 0.001367 \\
\hline \multirow{12}{*}{50} & \multirow{2}{*}{$\widehat{\alpha}_{1}$} & 1.74450 & 1.61835 & 1.45847 & 1.45849 & 1.45921 & 1.45991 & 1.45990 \\
\hline & & 0.466704 & 0.01490 & 0.002214 & 0.002210 & 0.002102 & 0.00108 & 0.00102 \\
\hline & \multirow[b]{2}{*}{$\widehat{\alpha}_{2}$} & 1.83449 & 1.01511 & 1.01534 & 1.01530 & 0.98625 & 0.98786 & 0.98788 \\
\hline & & 0.291776 & 0.000252 & 0.000258 & 0.000250 & 0.00044 & 0.00010 & 0.00010 \\
\hline & \multirow{2}{*}{$\widehat{\beta}_{1}$} & 0.65516 & 0.84292 & 0.83686 & 0.83680 & 0.80439 & 0.80339 & 0.80337 \\
\hline & & 0.024720 & 0.002230 & 0.002021 & 0.002021 & 0.00025 & 0.000211 & 0.000202 \\
\hline & \multirow{2}{*}{$\widehat{\beta}_{2}$} & 0.68191 & 0.54503 & 0.542902 & 0.542901 & 0.54978 & 0.54989 & 0.54980 \\
\hline & & 0.0174895 & 0.00223 & 0.00204 & 0.00203 & 0.00106 & 0.00010 & 0.00011 \\
\hline & \multirow{2}{*}{$\widehat{\delta}_{1}$} & 0.40616 & 0.40920 & 0.40795 & 0.40755 & 0.40769 & 0.40755 & 0.40750 \\
\hline & & 0.006272 & 0.00103 & 0.00057 & 0.00057 & 0.00057 & 0.00049 & 0.00040 \\
\hline & \multirow{2}{*}{$\widehat{R}(t)$} & 0.026996 & 0.037247 & 0.037346 & 0.037267 & 0.037312 & 0.037283 & 0.037266 \\
\hline & & 0.002229 & 0.001369 & 0.001361 & 0.001367 & 0.001364 & 0.001366 & 0.001367 \\
\hline \multirow{12}{*}{100} & \multirow{2}{*}{$\widehat{\alpha}_{1}$} & 1.12980 & 1.47760 & 1.4775 & 1.47759 & 1.47825 & 1.4780 & 1.4785 \\
\hline & & 0.037469 & 0.00051 & 0.00050 & 0.000490 & 0.00472 & 0.00462 & 0.00453 \\
\hline & \multirow{2}{*}{$\widehat{\alpha}_{2}$} & 2.39554 & 0.99279 & 0.99271 & 0.992701 & 0.99295 & 0.99290 & 0.99299 \\
\hline & & 0.1365480 & 0.00005 & 0.000051 & 0.00005 & 0.00049 & 0.00049 & 0.00049 \\
\hline & \multirow{2}{*}{$\widehat{\beta}_{1}$} & 1.04398 & 0.78192 & 0.78197 & 0.78199 & 0.78180 & 0.78183 & 0.781868 \\
\hline & & 0.0243720 & 0.000161 & 0.000160 & 0.000159 & 0.00158 & 0.00151 & 0.00150 \\
\hline & \multirow{2}{*}{$\widehat{\beta}_{2}$} & 0.61518 & 0.48740 & 0.48739 & 0.48749 & 0.48734 & 0.49735 & 0.49739 \\
\hline & & 0.0070329 & 0.00020 & 0.000161 & 0.000160 & 0.00193 & 0.001703 & 0.001702 \\
\hline & \multirow{2}{*}{$\widehat{\delta}_{1}$} & 0.453810 & 0.40697 & 0.4066 & 0.40087 & 0.40652 & 0.40647 & 0.40087 \\
\hline & & 0.0025615 & 0.000301 & 0.00029 & 0.00012 & 0.002730 & 0.00271 & 0.00012 \\
\hline & \multirow{2}{*}{$\widehat{R}(t)$} & 0.066993 & 0.037247 & 0.037346 & 0.037267 & 0.037312 & 0.037283 & 0.037266 \\
\hline & & 0.000519 & 0.001369 & 0.001361 & 0.001367 & 0.001364 & 0.001366 & 0.001367 \\
\hline
\end{tabular}

$$
\begin{aligned}
\delta_{1} & =0.40, \\
m_{1} & =22, \\
m_{2} & =34, \\
s_{1} & =25, \\
s_{2} & =26, \\
\sum_{j=1}^{s_{1}} \ln \left(1-\left(1+x_{1 i}\right)^{-\alpha_{1}}\right) & =-43.507, \\
\sum_{j=1}^{s_{2}} \ln \left(1-\left(1+x_{2 i}\right)^{-\alpha_{2}}\right) & =-43.773 .
\end{aligned}
$$

And we obtain the following required numerical quantities when $T=70$ :

$$
\begin{aligned}
\delta_{1} & =0.40, \\
m_{1} & =22, \\
m_{2} & =19, \\
s_{1} & =19, \\
s_{2} & =28, \\
\sum_{j=1}^{s_{1}} \ln \left(1-\left(1+x_{1 i}\right)^{-\alpha_{1}}\right) & =-33.195, \\
\sum_{j=1}^{s_{2}} \ln \left(1-\left(1+x_{2 i}\right)^{-\alpha_{2}}\right) & =-46.483 .
\end{aligned}
$$


TABLE 2: BEs, PRs, and reliability estimates using UP, IBeta, and gamma prior under LLF and SELF parameters $\left(\delta_{1}, \alpha_{1}, \alpha_{2}, \beta_{1}, \beta_{2}\right)(0.4,2,3$, $1.3,1.5)$ and $T=5$.

\begin{tabular}{|c|c|c|c|c|c|c|c|c|}
\hline \multirow{3}{*}{$n$} & \multirow{3}{*}{ Parameters } & \multirow{3}{*}{ MLEs } & \multicolumn{6}{|c|}{ Bayes estimates } \\
\hline & & & & SELF & & & LLF & \\
\hline & & & UP & IBeta & Gamma & UP & IBeta & Gamma \\
\hline \multirow{12}{*}{30} & \multirow{2}{*}{$\widehat{\alpha}_{1}$} & 2.320675 & 2.00356 & 2.00598 & 2.00398 & 2.00345 & 2.003098 & 2.00380 \\
\hline & & 0.601604 & 0.00001 & 0.00001 & 0.00001 & 0.00001 & 0.00000 & 0.00001 \\
\hline & \multirow{2}{*}{$\widehat{\alpha}_{2}$} & 1.764759 & 2.9989 & 2.9979 & 2.9999 & 2.99591 & 2.99567 & 2.99589 \\
\hline & & 0.60725 & 0.00087 & 0.00077 & 0.00073 & 0.00001 & 0.00001 & 0.00001 \\
\hline & \multirow{2}{*}{$\widehat{\beta}_{1}$} & 0.866185 & 1.23822 & 1.236987 & 1.23589 & 1.23519 & 1.23580 & 1.23518 \\
\hline & & 0.068320 & 0.00026 & 0.00020 & 0.00019 & 0.00026 & 0.00020 & 0.00026 \\
\hline & \multirow{2}{*}{$\widehat{\beta}_{2}$} & 1.36015 & 1.51891 & 1.51633 & 1.51478 & 1.51676 & 1.51674 & 1.51601 \\
\hline & & 0.098695 & 0.00026 & 0.00021 & 0.00019 & 0.00025 & 0.00029 & 0.00026 \\
\hline & \multirow{2}{*}{$\widehat{\delta}_{1}$} & 0.370755 & 0.42826 & 0.428555 & 0.42877 & 0.41089 & 0.41089 & 0.41089 \\
\hline & & 0.007937 & 0.00084 & 0.00012 & 0.00087 & 0.00012 & 0.00012 & 0.00012 \\
\hline & \multirow{2}{*}{$\widehat{R}(t)$} & 0.029982 & 0.037247 & 0.037346 & 0.037267 & 0.037312 & 0.037283 & 0.037266 \\
\hline & & 0.001955 & 0.001369 & 0.001361 & 0.001367 & 0.001364 & 0.001366 & 0.001367 \\
\hline \multirow{12}{*}{50} & \multirow{2}{*}{$\widehat{\alpha}_{1}$} & 1.63092 & 2.00310 & 2.00315 & 2.00300 & 2.0031 & 2.003001 & 2.00307 \\
\hline & & 0.196138 & 0.00001 & 0.00001 & 0.00001 & 0.00001 & 0.00000 & 0.00001 \\
\hline & \multirow[b]{2}{*}{$\widehat{\alpha}_{2}$} & 1.986596 & 2.9959 & 2.9969 & 2.9909 & 2.99590 & 2.99509 & 2.99599 \\
\hline & & 0.191953 & 0.00087 & 0.00077 & 0.00073 & 0.00001 & 0.00001 & 0.00001 \\
\hline & \multirow{2}{*}{$\widehat{\beta}_{1}$} & 1.55010 & 1.2354 & 1.23674 & 1.23504 & 1.235103 & 1.2358 & 1.23511 \\
\hline & & 0.124060 & 0.00026 & 0.00020 & 0.00019 & 0.00026 & 0.00020 & 0.00026 \\
\hline & \multirow{2}{*}{$\widehat{\beta}_{2}$} & 1.375517 & 1.51610 & 1.51610 & 1.51401 & 1.51698 & 1.51674 & 1.51601 \\
\hline & & 0.0653742 & 0.00026 & 0.00021 & 0.00019 & 0.00025 & 0.00029 & 0.00026 \\
\hline & \multirow{2}{*}{$\widehat{\delta}_{1}$} & 0.40352 & 0.40920 & 0.40795 & 0.40755 & 0.40769 & 0.40755 & 0.40750 \\
\hline & & 0.005197 & 0.0010 & 0.00057 & 0.00057 & 0.00057 & 0.00049 & 0.00041 \\
\hline & \multirow{2}{*}{$\widehat{R}(t)$} & 0.026996 & 0.037247 & 0.037346 & 0.037267 & 0.037312 & 0.037283 & 0.037266 \\
\hline & & 0.002229 & 0.001369 & 0.001361 & 0.001367 & 0.001364 & 0.001366 & 0.001367 \\
\hline \multirow{12}{*}{100} & \multirow{2}{*}{$\widehat{\alpha}_{1}$} & 1.630920 & 2.00310 & 2.00315 & 2.00300 & 1.3590 & 1.30142 & 1.2901 \\
\hline & & 0.1961383 & 0.00001 & 0.0000 & 0.00003 & 0.02675 & 0.04129 & 0.04720 \\
\hline & \multirow{2}{*}{$\widehat{\alpha}_{2}$} & 1.986596 & 2.9959 & 2.9969 & 2.9909 & 0.96086 & 0.95680 & 0.95581 \\
\hline & & 0.1919537 & 0.00085 & 0.00077 & 0.00073 & 0.00188 & 0.00187 & 0.00201 \\
\hline & \multirow{2}{*}{$\widehat{\beta}_{1}$} & 1.550108 & 1.2354 & 1.23674 & 1.23504 & 0.65693 & 0.68702 & 0.69703 \\
\hline & & 0.1240605 & 0.000262 & 0.000202 & 0.000192 & 0.00401 & 0.00424 & 0.00450 \\
\hline & \multirow{2}{*}{$\widehat{\beta}_{2}$} & 1.375517 & 1.5161 & 1.516101 & 1.51401 & 0.43707 & 0.43454 & 0.43331 \\
\hline & & 0.065374 & 0.00026 & 0.00021 & 0.00019 & 0.004021 & 0.004342 & 0.00450 \\
\hline & \multirow{2}{*}{$\widehat{\delta}_{1}$} & 0.403526 & 0.40920 & 0.40795 & 0.40755 & 0.40769 & 0.40755 & 0.40750 \\
\hline & & 0.0051975 & 0.0010 & 0.00057 & 0.00057 & 0.00057 & 0.00049 & 0.00041 \\
\hline & \multirow{2}{*}{$\widehat{R}(t)$} & 0.066993 & 0.037247 & 0.037346 & 0.037267 & 0.037312 & 0.037283 & 0.037266 \\
\hline & & 0.000519 & 0.001369 & 0.001361 & 0.001367 & 0.001364 & 0.001366 & 0.001367 \\
\hline
\end{tabular}

$$
\begin{aligned}
\delta_{1} & =0.40, \\
m_{1} & =22, \\
m_{2} & =34, \\
s_{1} & =21, \\
s_{2} & =22, \\
\sum_{j=1}^{s_{1}} \ln \left(1-\left(1+x_{1 i}\right)^{-\alpha_{1}}\right) & =-37.657, \\
\sum_{j=1}^{s_{2}} \ln \left(1-\left(1+x_{2 i}\right)^{-\alpha_{2}}\right) & =-34.689 .
\end{aligned}
$$

To analyze the data of the burning velocity of chemical materials, we consider three different censoring times. The data thus acquired are used to get MLEs and BEs of the parameter of the IKum mixture model that are given in Table 4. Bayes estimates are obtained assuming uniform, gamma, and inverse beta priors. However, two loss functions SELF and LLF are used for Bayesian estimation. It is found that MLEs are a bit lower than Bayes estimates. The mixture model comprises five parameters whose all four parameters are shape parameters except mixing weight. Mixing weight which is considered 0.40 in the mixture data is almost ideally estimated. Shape parameter $\alpha$ is estimated to be about on average 1.5 to $1.8 \mathrm{~cm} / \mathrm{sec}$ for the first component, and for the second component density, it ranges from almost 0.90 to $1.00 \mathrm{~cm} / \mathrm{s}$.

3.3. Simulation Study of Real Data. In this section, using the estimates of the real dataset, we determine the estimates through a simulation study. It is observed that the Bayes estimators obtained through simulation are very close to the true values of estimators. One can easily observe that the suitable prior for these data is gamma prior and the best loss function is LLF because they provide less posterior risk. Results are given in Table 5. 


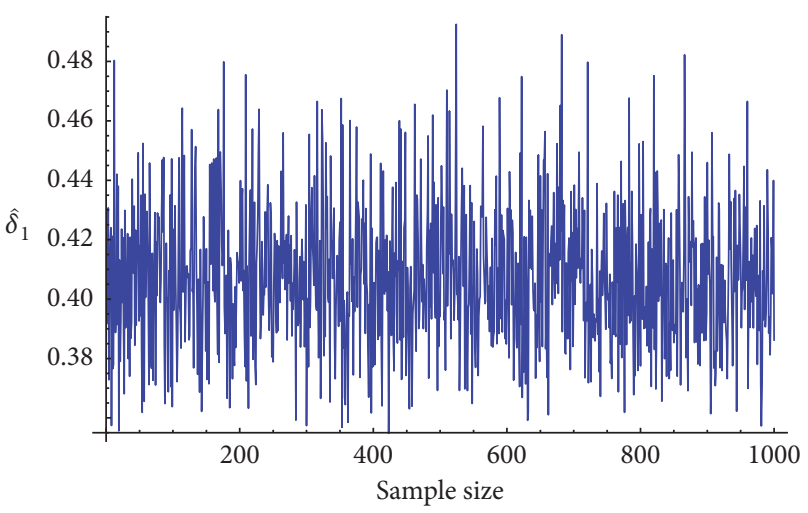

(a)

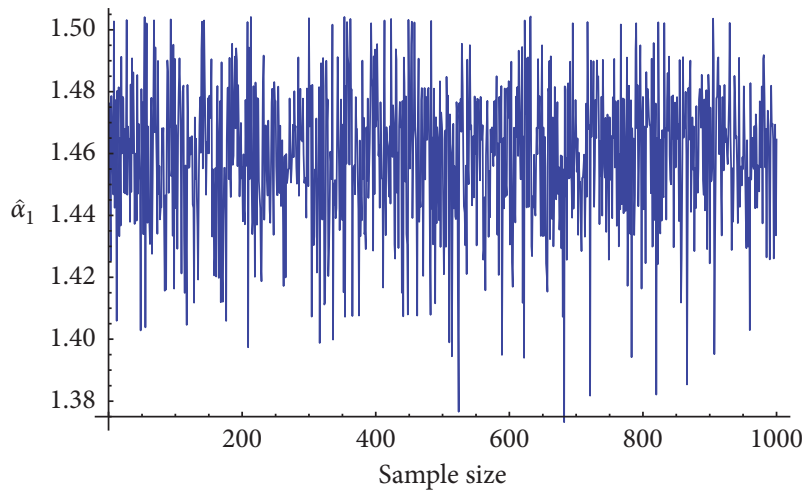

(c)

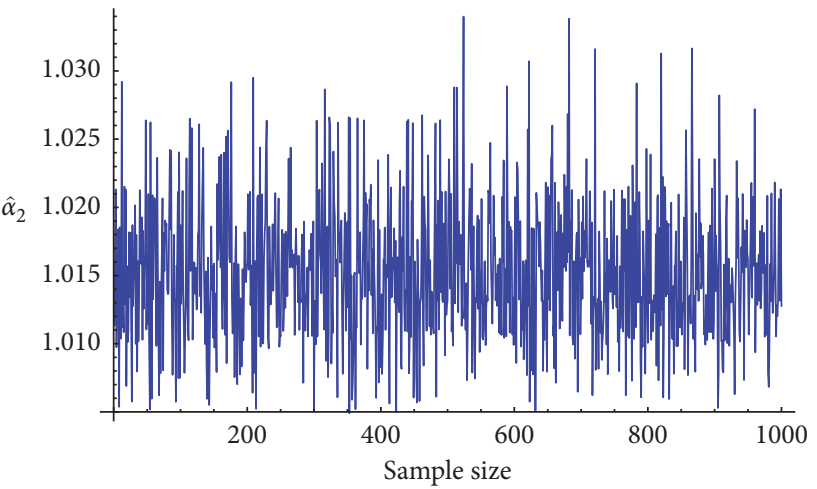

(e)

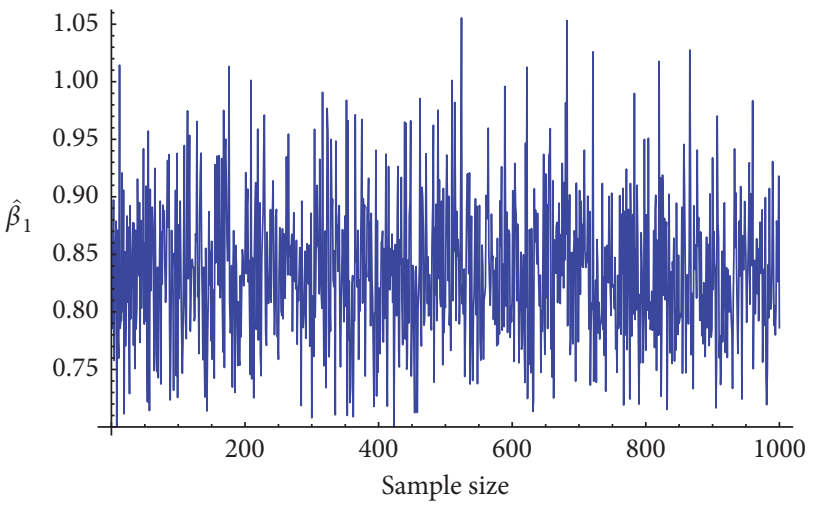

(g)

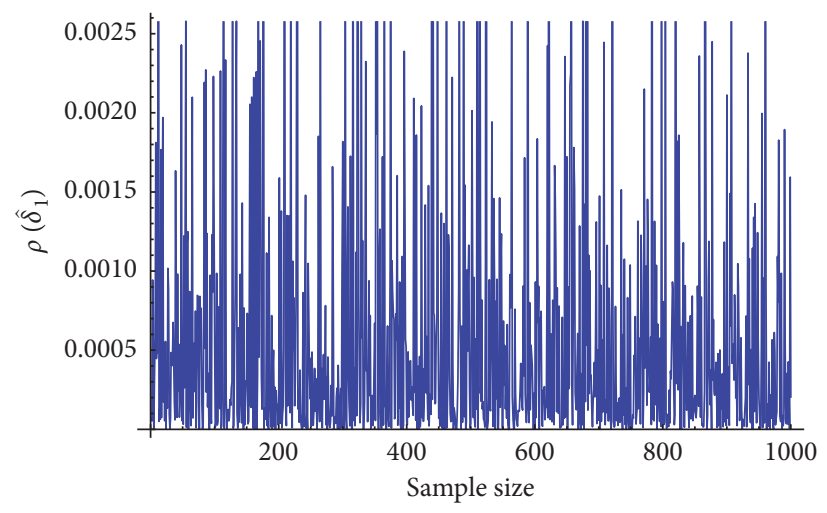

(b)

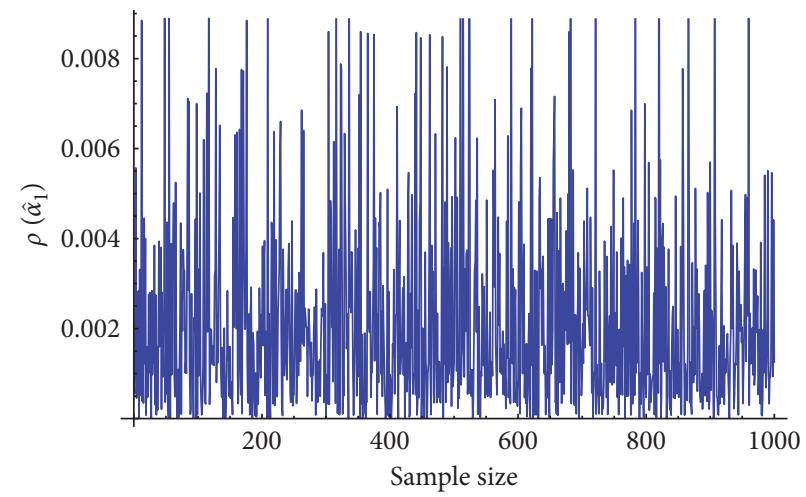

(d)

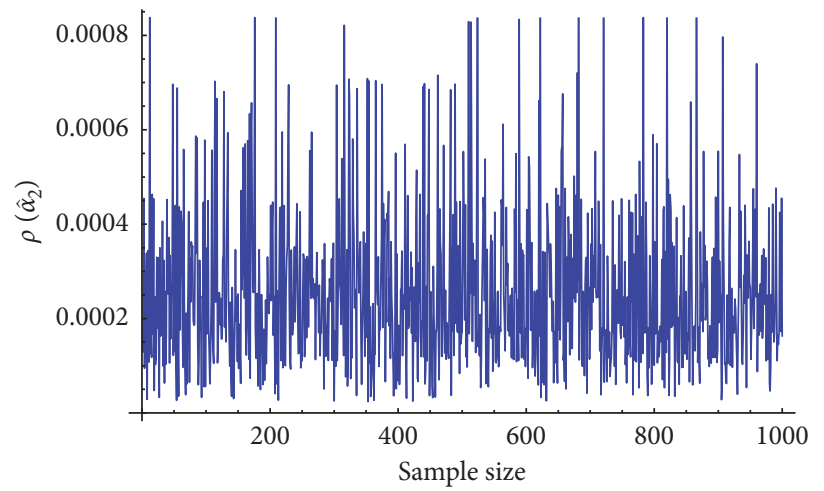

(f)

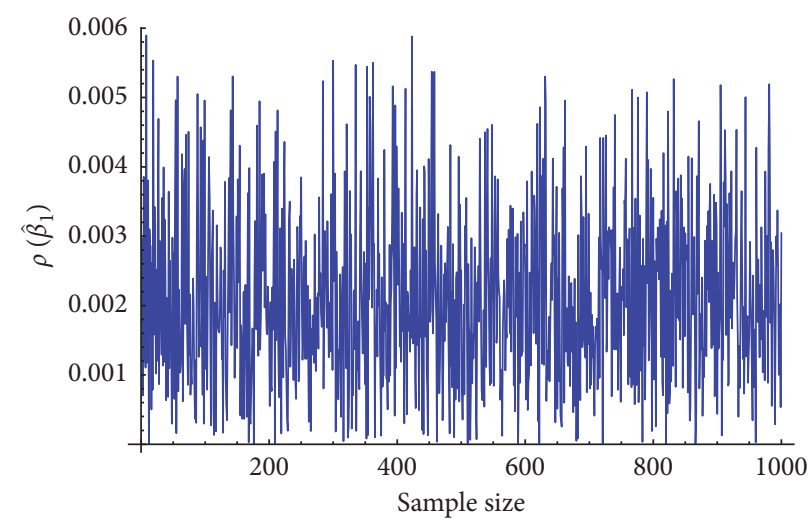

(h)

Figure 1: Continued. 


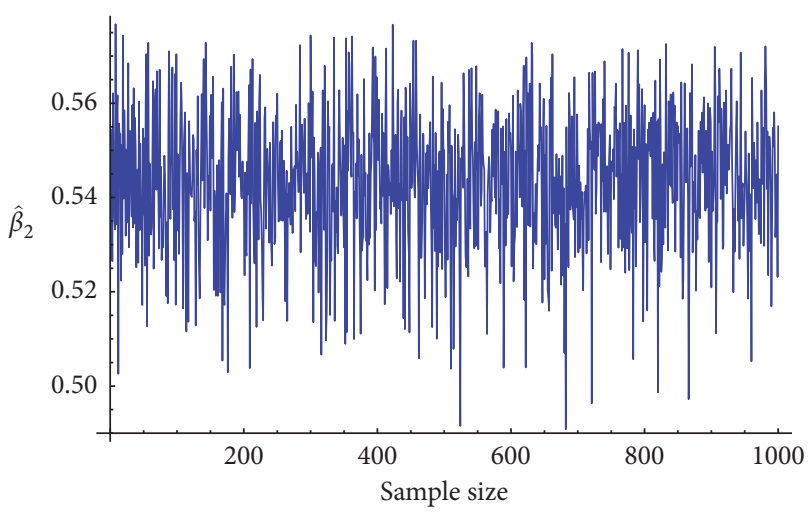

(i)

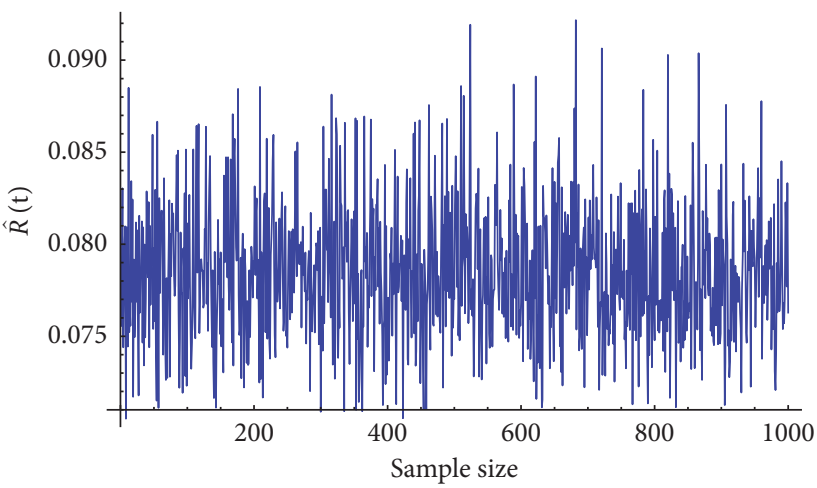

$(\mathrm{k})$

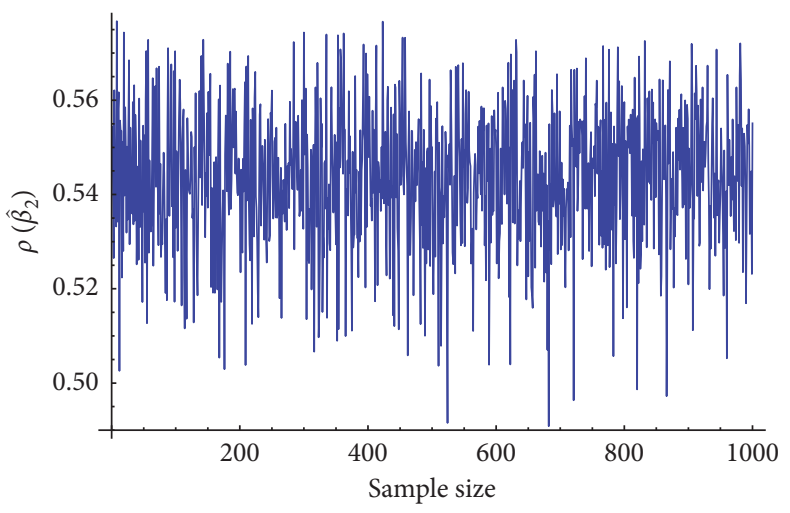

(j)

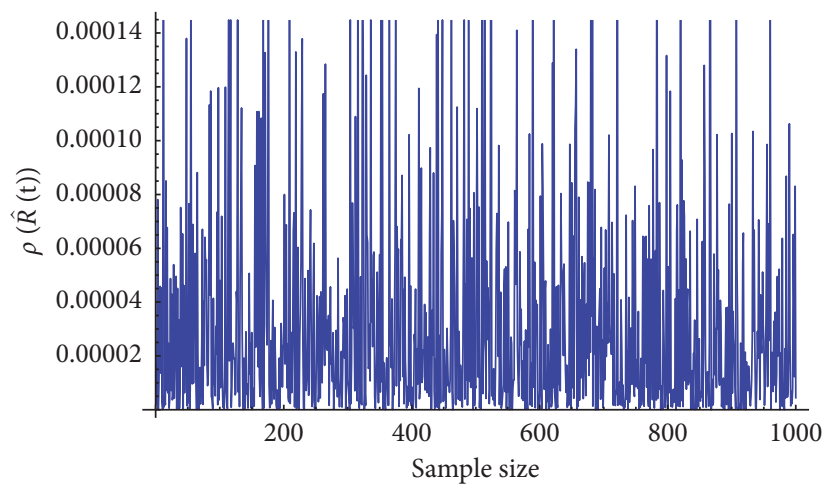

(1)

FIGURE 1: Graphical representation of the Bayes estimators, posterior risk, and reliability function of the IKum mixture model based on simulation studies.

TABLe 3: Real dataset of the burning velocity of chemical materials.

\begin{tabular}{|c|c|c|c|c|c|c|c|c|c|c|c|c|c|}
\hline 68 & 61 & 64 & 55 & 51 & 68 & 44 & 50 & 82 & 60 & 89 & 61 & 54 & 166 \\
\hline 66 & 50 & 87 & 48 & 42 & 58 & 46 & 67 & 46 & 46 & 44 & 48 & 56 & 47 \\
\hline 54 & 47 & 89 & 38 & 108 & 46 & 40 & 44 & 312 & 41 & 31 & 40 & 41 & 40 \\
\hline 56 & 45 & 43 & 46 & 46 & 46 & 46 & 52 & 58 & 82 & 71 & 48 & 39 & 41 \\
\hline
\end{tabular}

TABLe 4: BEs and their PRs and reliability estimate using UP and IP (inverse beta and gamma) under LLF and SELF.

\begin{tabular}{|c|c|c|c|c|c|c|c|}
\hline \multirow{3}{*}{ Parameters } & \multirow{3}{*}{ MLEs } & \multicolumn{6}{|c|}{ Bayes estimates } \\
\hline & & \multicolumn{3}{|c|}{ SELF } & \multicolumn{3}{|c|}{ LLF } \\
\hline & & UP & IBeta & Gamma & UP & IBeta & Gamma \\
\hline \multicolumn{8}{|c|}{$T=85, n=56$} \\
\hline \multirow[b]{2}{*}{$\widehat{\alpha}_{1}$} & 1.37051 & 1.83988 & 1.83980 & 1.85330 & 1.78270 & 1.78143 & 1.54997 \\
\hline & 0.08924 & 0.11546 & 0.11540 & 0.11538 & 0.01123 & 0.01120 & 0.00301 \\
\hline \multirow{2}{*}{$\widehat{\alpha}_{2}$} & 0.59800 & 0.92089 & 0.92087 & 0.92880 & 0.92446 & 0.92450 & 0.99108 \\
\hline & 0.01446 & 0.00627 & 0.00626 & 0.00624 & 0.00034 & 0.00024 & 0.00007 \\
\hline \multirow{2}{*}{$\widehat{\beta}_{1}$} & 0.57709 & 0.85566 & 0.85560 & 0.86400 & 0.91727 & 0.91769 & 0.81050 \\
\hline & 0.013332 & 0.00301 & 0.00309 & 0.00309 & 0.00025 & 0.00021 & 0.00009 \\
\hline \multirow{2}{*}{$\widehat{\beta}_{2}$} & 0.70302 & 0.51546 & 0.51544 & 0.57839 & 0.64110 & 0.64105 & 0.52460 \\
\hline & 0.01606 & 0.00025 & 0.00024 & 0.00023 & 0.00055 & 0.00055 & 0.00035 \\
\hline \multirow{2}{*}{$\widehat{\delta}_{1}$} & 0.44828 & 0.42232 & 0.42202 & 0.42709 & 0.42662 & 0.42618 & 0.40190 \\
\hline & 0.00446 & 0.00048 & 0.00048 & 0.00041 & 0.00010 & 0.00011 & 0.00001 \\
\hline \multirow{2}{*}{$\widehat{R}(t)$} & 0.94381 & 0.99511 & 0.99562 & 0.99403 & 0.99675 & 0.99820 & 0.99862 \\
\hline & 0.00196 & 0.00001 & 0.00001 & 0.00006 & 0.00012 & 0.00013 & 0.00012 \\
\hline
\end{tabular}


TABLE 4: Continued.

\begin{tabular}{|c|c|c|c|c|c|c|c|}
\hline \multirow{3}{*}{ Parameters } & \multirow{3}{*}{ MLEs } & \multicolumn{6}{|c|}{ Bayes estimates } \\
\hline & & \multicolumn{3}{|c|}{ SELF } & \multicolumn{3}{|c|}{ LLF } \\
\hline & & UP & IBeta & Gamma & UP & IBeta & Gamma \\
\hline \multicolumn{8}{|c|}{$T=70, n=56$} \\
\hline \multirow{2}{*}{$\widehat{\alpha}_{1}$} & 1.06862 & 1.83988 & 1.80750 & 1.81851 & 1.78271 & 1.78120 & 1.54971 \\
\hline & 0.06010 & 0.11546 & 0.09458 & 0.10148 & 0.01123 & 0.01120 & 0.00305 \\
\hline \multirow{2}{*}{$\widehat{\alpha}_{2}$} & 1.30658 & 0.92089 & 0.92659 & 0.93250 & 0.92446 & 0.92449 & 0.99104 \\
\hline & 0.06097 & 0.00627 & 0.00539 & 0.00455 & 0.00034 & 0.00023 & 0.00006 \\
\hline \multirow{2}{*}{$\widehat{\beta}_{1}$} & 0.57246 & 0.85566 & 0.84716 & 0.85708 & 0.91727 & 0.91766 & 0.81049 \\
\hline & 0.01725 & 0.00309 & 0.00220 & 0.00325 & 0.00026 & 0.00021 & 0.00009 \\
\hline \multirow{2}{*}{$\widehat{\beta}_{2}$} & 0.59864 & 0.51546 & 0.51452 & 0.57248 & 0.64110 & 0.64101 & 0.52459 \\
\hline & 0.01280 & 0.00024 & 0.00021 & 0.00525 & 0.00055 & 0.00055 & 0.00035 \\
\hline \multirow{2}{*}{$\widehat{\delta}_{1}$} & 0.40425 & 0.42232 & 0.42029 & 0.42469 & 0.42662 & 0.42612 & 0.40197 \\
\hline & 0.00520 & 0.00048 & 0.00041 & 0.00060 & 0.00011 & 0.00011 & 0.00001 \\
\hline \multirow{2}{*}{$\widehat{R}(t)$} & 0.93676 & 0.99516 & 0.99516 & 0.99412 & 0.99822 & 0.99820 & 0.99862 \\
\hline & 0.00223 & 0.00001 & 0.00001 & 0.00002 & 0.00003 & 0.00003 & 0.00074 \\
\hline \multicolumn{8}{|c|}{$T=65, n=56$} \\
\hline \multirow{2}{*}{$\widehat{\alpha}_{1}$} & 1.18110 & 1.6711 & 1.67010 & 1.67012 & 1.52543 & 1.52441 & 1.52420 \\
\hline & 0.06643 & 0.02896 & 0.02899 & 0.02894 & 0.00115 & 0.00116 & 0.00100 \\
\hline \multirow{2}{*}{$\widehat{\alpha}_{2}$} & 1.02660 & 0.95929 & 0.95923 & 0.95892 & 0.99544 & 0.99546 & 0.99540 \\
\hline & 0.04790 & 0.00162 & 0.00626 & 0.00160 & 0.00002 & 0.00002 & 0.00002 \\
\hline \multirow{2}{*}{$\widehat{\beta}_{1}$} & 0.56466 & 0.82492 & 0.82492 & 0.82482 & 0.80514 & 0.80543 & 0.80501 \\
\hline & 0.01518 & 0.00062 & 0.00309 & 0.00062 & 0.00005 & 0.00006 & 0.00005 \\
\hline \multirow{2}{*}{$\widehat{\beta}_{2}$} & 0.63268 & 0.50818 & 0.50819 & 0.50812 & 0.51218 & 0.51267 & 0.51215 \\
\hline & 0.01819 & 0.00006 & 0.00023 & 0.00006 & 0.00017 & 0.00020 & 0.00010 \\
\hline \multirow{2}{*}{$\widehat{\delta}_{1}$} & 0.48837 & 0.41121 & 0.41189 & 0.41120 & 0.40130 & 0.40124 & 0.40103 \\
\hline & 0.00581 & 0.00012 & 0.00012 & 0.00012 & 0.00002 & 0.00002 & 0.00001 \\
\hline \multirow{2}{*}{$\widehat{R}(t)$} & 0.93409 & 0.99653 & 0.99654 & 0.99489 & 0.99663 & 0.99693 & 0.99899 \\
\hline & 0.00052 & 0.00000 & 0.00000 & 0.00001 & 0.00000 & 0.00000 & 0.00000 \\
\hline
\end{tabular}

TABle 5: BEs, PRs, MLEs with MSEs, and reliability estimates using UP and IP (inverse beta and gamma) under LLF and SELF.

\begin{tabular}{|c|c|c|c|c|c|c|c|}
\hline \multirow{3}{*}{ Parameters } & \multirow{3}{*}{ MLEs } & \multicolumn{6}{|c|}{ Bayes estimates } \\
\hline & & \multicolumn{3}{|c|}{ SELF } & \multicolumn{3}{|c|}{ LLF } \\
\hline & & UP & IBeta & Gamma & UP & IBeta & Gamma \\
\hline \multirow{2}{*}{$\widehat{\alpha}_{1}$} & 2.32066 & 1.51740 & 1.48950 & 1.85330 & 1.78270 & 1.78120 & 1.54970 \\
\hline & 0.60160 & 0.00834 & 0.01369 & 0.11538 & 0.01123 & 0.01120 & 0.00304 \\
\hline \multirow{2}{*}{$\widehat{\alpha}_{2}$} & 1.76476 & 1.01512 & 1.01593 & 0.92883 & 0.92446 & 0.92449 & 0.99104 \\
\hline & 0.60725 & 0.00071 & 0.00075 & 0.00624 & 0.00034 & 0.00023 & 0.00009 \\
\hline \multirow{2}{*}{$\widehat{\beta}_{1}$} & 0.86619 & 0.93457 & 0.93516 & 0.86400 & 0.91727 & 0.91766 & 0.81049 \\
\hline & 0.06832 & 0.00122 & 0.00212 & 0.00309 & 0.00025 & 0.00021 & 0.00009 \\
\hline \multirow{2}{*}{$\widehat{\beta}_{2}$} & 1.36015 & 0.54724 & 0.56054 & 0.57839 & 0.64110 & 0.64101 & 0.52459 \\
\hline & 0.09869 & 0.00127 & 0.00212 & 0.00023 & 0.00055 & 0.00055 & 0.00035 \\
\hline \multirow{2}{*}{$\widehat{\delta}_{1}$} & 0.37076 & 0.45179 & 0.45830 & 0.42709 & 0.42662 & 0.42612 & 0.40197 \\
\hline & 0.00794 & 0.00420 & 0.00932 & 0.00048 & 0.00012 & 0.00011 & 0.00002 \\
\hline \multirow{2}{*}{$\widehat{R}(t)$} & 0.94176 & 0.95196 & 0.98027 & 0.98909 & 0.95199 & 0.98027 & 0.99693 \\
\hline & 0.00196 & 0.000004 & 0.00000 & 0.00000 & 0.00000 & 0.00000 & 0.00000 \\
\hline
\end{tabular}

\section{Conclusion}

In this paper, we conduct a Bayesian estimation of the unknown parameters and reliability function of the inverted Kumaraswamy mixture model under type 1 right censoring. For the choice of different sample sizes, $n=30,50$, and 100 are taken to perform a simulation study. It is observed as the sample size increases the parameters converge to their true parametric value. And it is also noted that LLF is found to be the best loss function assuming gamma prior because it has a less posterior risk.
Bayes estimates are found overestimated for some values and underestimated for few values. From the real dataset, it is observed that as the censoring times $T=85,70$, and 65 decrease, the posterior risk also decreases respectively. The BEs $\widehat{\alpha}_{1}, \widehat{\alpha}_{2}$, $\widehat{\beta}_{1}$, and $\widehat{\beta}_{2}$ represent the mean value of the burning velocity of the chemical material. And $\widehat{R}(t)$ represents the reliability of the estimates. Simulation of real data is carried out to compare the parametric values. Graphical representation of parameters is also presented by taking the number of iterations on the $x$-axis and different parametric values on the $y$-axis. From these graphs, we 
come up with almost the same results of Bayes estimates. Bayes estimates are found better than MLEs as PRs are smaller as compared to MSEs.

\section{Appendix}

\section{A. Numerical Results of Simulation Study}

BEs, PRs, and reliability estimates using UP, IBeta, and gamma prior under LLF and SELF for parameters $\left(\delta_{1}, \alpha_{1}\right.$, $\left.\alpha_{2}, \beta_{1}, \beta_{2}\right)(0.4,1.5,1,0.8,0.5)$ and $T=5$ and $(0.4,2,3,1.3$, $1.5)$ and $T=5$ are shown.

\section{B. Derivation for the Elements of Lindley's Approximation}

Taking logarithm of posterior density given in equation (8)

$$
\begin{aligned}
Q(\Theta \mid x)= & \ln p(\Theta \mid x) \propto s_{1} \ln \delta_{1}+s_{2} \ln \left(1-\delta_{1}\right)+\left(a_{1}+s_{1}-1\right) \ln \alpha_{1}+\left(a_{2}+s_{2}-1\right) \ln \alpha_{2} \\
& +\left(c_{1}+s_{1}-1\right) \ln \beta_{1}+\left(c_{2}+s_{2}-1\right) \ln \beta_{2}-b_{1} \alpha_{1}-b_{2} \alpha_{2}-c_{1} \beta_{1}-c_{2} \beta_{2}-\left(\alpha_{1}-1\right) \sum_{j=1} \ln \left(1+x_{1 j}\right) \\
& -\left(\alpha_{2}-1\right) \sum_{j=1}^{s_{2}} \ln \left(1+x_{2 j}\right)+\left(\beta_{1}-1\right) \sum_{j=1}^{s_{1}} \ln \left(1-\left(1+x_{1 j}\right)^{-\alpha_{1}}\right)+\left(\beta_{2}-1\right) \sum_{j=1}^{s_{2}} \ln \left(1-\left(1+x_{2 j}\right)^{-\alpha_{2}}\right)+ \\
& (m-s) \ln \left(1-\delta_{1}\left(1-\left(1+t_{o}\right)^{-\alpha_{1}}\right)-\left(1-\delta_{1}\right)\left(1-\left(1+t_{o}\right)^{-\alpha_{1}}\right)\right) .
\end{aligned}
$$

From equations (B.1) and (10), the elements $Q_{i j s}, Q_{i j s}=$ $Q_{i s j}=Q_{s i j}=Q_{j i s}$ and $t_{o}=T$ for $i, j, s=1,2, \ldots, 5$ are derived as follows:

$$
\begin{aligned}
& \left\{\begin{array}{l}
\varphi_{i}=(1+T)^{-\alpha_{i}} \ln (1+T), \\
\psi_{i}=\sum_{j=1}^{m-s} \frac{\left(1+x_{i j}\right)^{-\alpha i} \ln \left(1+x_{j i}\right)}{\left(1-\left(1+x_{1 i}\right)^{-\alpha_{i}}\right)} \\
k_{i}=-F_{i} \ln \left(1-(1+T)^{-\alpha_{i}}\right), \\
l_{i}=\frac{\beta_{i} F_{i} \varphi_{i}}{\left(1-(1+T)^{-\alpha_{i}}\right)}, \\
m_{i}=\frac{\delta_{i} F_{i} \varphi_{i}}{\left(1-(1+T)^{-\alpha_{i}}\right)\left(1+\beta_{i} \ln \left(1-(1+T)^{-\alpha_{i}}\right)\right)}, \\
\theta_{i}=\frac{\delta_{i} F_{i} \varphi_{i} \beta_{i} \ln (1+T)-\left(\beta_{i}-1\right) \varphi_{i}}{\left(1-(1+T)^{-\alpha_{i}}\right)^{2}} \\
z_{i}=-\delta_{i}\left(\ln \left(1-(1+T)^{-\alpha_{i}}\right)\right)^{2} F_{i},
\end{array} \quad i=1,2,\right. \\
& Q_{11}=-\left[\frac{s_{1}}{\delta_{1}^{2}}+\frac{s_{2}}{\left(1-\delta_{1}\right)^{2}}+\frac{(m-s)\left(F_{2}-F_{1}\right)^{2}}{R^{2}}\right] \text {, } \\
& Q_{12}=\frac{(m-s) F_{1} \varphi_{1} \beta_{1}\left(p_{1}\left(F_{2}-F_{1}\right)-R\right)}{\left(1-(1+T)^{-\alpha_{1}}\right) R^{2}}, \\
& Q_{13}=\frac{(m-s) F_{2} \varphi_{2} \beta_{2}\left(\left(1-p_{1}\right)\left(F_{2}-F_{1}\right)+R\right)}{\left(1-(1+T)^{-\alpha_{2}}\right) R^{2}}, \\
& Q_{14}=\frac{(m-s) F_{1} \ln \left(1-(1+T)^{-\alpha_{1}}\right)\left(\left(F_{2}-F_{1}\right) p_{1}-R\right)}{R^{2}}, \\
& Q_{15}=\frac{(m-s) F_{2} \ln \left(1-(1+T)^{-\alpha_{2}}\right)\left(\left(F_{2}-F_{1}\right)\left(1-p_{1}\right)+R\right)}{R^{2}},
\end{aligned}
$$




$$
\begin{aligned}
& Q_{22}=-\frac{s_{1}}{\alpha_{1}^{2}}-\left(\beta_{1}-1\right) \psi_{11}+\psi_{1}^{2}+\frac{(m-s) \delta_{1} \beta_{1} \varphi_{1} F_{1}}{\left(1-(1+T)^{\alpha_{1}}\right) R}\left(\ln (1+T)-\frac{\left(\beta_{1}-1\right) \varphi_{1}}{\left(1-(1+T)^{\alpha_{1}}\right)}-\frac{\delta_{1} \beta_{1} \varphi_{1} F_{1}}{\left(1-(1+T)^{\alpha_{1}}\right) R}\right), \\
& Q_{23}=Q_{32}=\frac{(m-s) \delta_{1}\left(1-\delta_{1}\right) \beta_{1} \beta_{2} \varphi_{1} \varphi_{2} F_{1} F_{2}}{\left(1-(1+T)^{-\alpha_{1}}\right)\left(1-(1+T)^{-\alpha_{2}}\right) R^{2}} \\
& Q_{24}=Q_{42}=\psi_{1}-\frac{(m-s) \delta_{1} \varphi_{1} F_{1}}{\left(1-(1+T)^{-\alpha_{2}}\right) R^{2}}\left(\frac{1}{\left(1-(1+T)^{-\alpha_{1}}\right)}+\frac{\ln \left(1-(1+T)^{-\alpha_{1}}\right) \delta_{1} \beta_{1} F_{1}}{R}+\ln \left(1-(1+T)^{-\alpha_{1}}\right) \beta_{1}\right) \text {, } \\
& Q_{25}=Q_{52}=\frac{-(m-s) p_{1}\left(1-\delta_{1}\right) \beta_{1} \varphi_{1} F_{1} F_{2} \ln \left(1-(1+T)^{-\alpha_{2}}\right)}{\left(1-(1+T)^{-\alpha_{1}}\right) R^{2}} \text {, } \\
& Q_{33}=-\frac{s_{2}}{\alpha_{2}^{2}}-\left(\beta_{2}-1\right)\left(\psi_{2}^{2}+\psi_{22}\right)-\frac{(m-s)\left(1-\delta_{1}\right) \beta_{2} \varphi_{2} F_{2}}{\left(1-(1+T)^{-\alpha_{2}}\right) R}\left(\ln (1+T)+\frac{\left(\beta_{2}-1\right) \varphi_{2}}{\left(1-(1+T)^{-\alpha_{2}}\right)}+\frac{\left(1-\delta_{1}\right) \beta_{2} \varphi_{2} F_{2}}{\left(1-(1+T)^{-\alpha_{2}}\right) R}\right), \\
& Q_{34}=Q_{43}=\frac{(m-s) \delta_{1}\left(1-\delta_{1}\right) \beta_{2} \varphi_{2} F_{1} F_{2} \ln \left(1-(1+T)^{-\alpha_{2}}\right)}{\left(1-(1+T)^{-\alpha_{1}}\right)\left(1-(1+T)^{-\alpha_{2}}\right) R^{2}}, \\
& Q_{35}=Q_{53}=-\frac{(m-s)\left(1-\delta_{1}\right) \varphi_{2} F_{2}}{\left(1-(1+T)^{-\alpha_{2}}\right) R}\left(1+\beta_{2} \ln \left(1-(1+T)^{-\alpha_{2}}\right)+\frac{\left(1-\delta_{1}\right) \beta_{2} F_{2} \ln \left(1-(1+T)^{-\alpha_{2}}\right)}{R}\right) \text {, } \\
& Q_{44}=-\frac{s_{1}}{\beta_{1}^{2}}-\frac{(m-s) \delta_{1} F_{2} \ln \left(1-(1+T)^{-\alpha_{1}}\right)}{R}\left(1+\frac{\delta_{1} F_{2}}{R}\right) \text {, } \\
& Q_{45}=Q_{54}=\frac{-(m-s) \delta_{1}\left(1-\delta_{1}\right) F_{1} F_{2} \ln \left(1-(1+T)^{-\alpha_{1}}\right) \ln \left(1-(1+T)^{-\alpha_{2}}\right)}{R^{2}} \text {, } \\
& Q_{55}=-\frac{s_{2}}{\beta_{2}^{2}}-\frac{(m-s)\left(1-\delta_{1}\right) F_{2}\left(\ln \left(1-(1+T)^{-\alpha 2}\right)^{2}\right)}{R}\left(\frac{\left(1-\delta_{1}\right) F_{2}}{R}+1\right) \text {, }
\end{aligned}
$$$$
Q_{111}=\left(\frac{2 s_{1}}{\delta_{1}^{3}}-\frac{2 s_{2}}{\left(1-\delta_{1}\right)^{3}}+\frac{2(m-s)\left(F_{2}-F_{1}\right)^{3}}{R^{3}}\right),
$$$$
Q_{112}=Q_{112}=\frac{2(m-s)\left(F_{2}-F_{1}\right) F_{1} \beta_{1} \varphi_{1}}{\left(1-(1+T)^{-\alpha_{1}}\right) R^{2}}\left(1-\frac{\delta_{1}\left(F_{2}-F_{1}\right)}{R}\right) \text {, }
$$$$
Q_{113}=Q_{131}=-\frac{2(m-s)\left(F_{2}-F_{1}\right) F_{2} \beta_{2} \varphi_{2}}{\left(1-(1+T)^{-\alpha_{2}}\right) R^{2}}\left(1+\frac{\left(1-\delta_{1}\right)\left(F_{2}-F_{1}\right)}{R}\right) \text {, }
$$$$
Q_{114}=Q_{141}=\frac{2(m-s)\left(F_{2}-F_{1}\right) F_{1} \ln \left(1-(1+T)^{-\alpha_{1}}\right)}{R^{2}}\left(1-\frac{\delta_{1}\left(F_{2}-F_{1}\right)}{R}\right) \text {, }
$$$$
Q_{115}=Q_{151}=\frac{2(m-s)\left(F_{2}-F_{1}\right) F_{2} \ln \left(1-(1+T)^{-\alpha_{2}}\right)}{R^{2}}\left(1-\frac{\left(1-\delta_{1}\right)\left(F_{2}-F_{1}\right)}{R}\right) \text {, }
$$

$Q_{122}=Q_{211}=\frac{(m-s) F_{1} \beta_{1} \varphi_{1}}{\left(1-(1+T)^{-\alpha_{1}}\right) R}\left(\begin{array}{c}-\frac{\delta_{1}\left(F_{2}-F_{1}\right) \ln (1+T)}{R}+\ln (1+T)+\frac{\beta_{1} \varphi_{1} \delta_{1}\left(F_{2}-F_{1}\right) \ln (1+T)}{\left(1-(1+T)^{-\alpha_{1}}\right) R}-\frac{\left(\beta_{1}-1\right) \varphi_{1}}{\left(1-(1+T)^{-\alpha_{1}}\right)} \\ +\frac{2 \delta_{1}^{2} \beta_{1} \varphi_{1}\left(F_{2}-F_{1}\right) F_{1}}{\left(1-(1+T)^{-\alpha_{1}}\right) R^{2}}-\frac{2 \beta_{1} \varphi_{1} \delta_{1} F_{1}}{\left(1-(1+T)^{-\alpha_{1}}\right) R}\end{array}\right)$,

$Q_{123}=Q_{132}=Q_{312}=\frac{(m-s) F_{1} F_{2} \beta_{1} \beta_{2} \varphi_{2} \varphi_{1}}{\left(1-(1+T)^{-\alpha_{1}}\right)\left(1-(1+T)^{-\alpha_{2}}\right) R^{2}}\left(\frac{2 \delta_{1}\left(F_{2}-F_{1}\right)\left(1-\delta_{1}\right)}{R}+2 \delta_{1}-1\right)$,

$Q_{124}=Q_{142}=Q_{412}=\frac{(m-s) F_{1} \varphi_{1}}{\left(1-(1+T)^{-\alpha_{1}}\right) R^{2}}\left(\begin{array}{c}\frac{\delta_{1}\left(F_{2}-F_{1}\right)}{R}-1+\frac{2 \delta_{1}^{2} F_{1} \beta_{1} \ln \left(1-(1+T)^{-\alpha_{1}}\right)}{R^{2}}-\frac{2 \delta_{1} F_{1} \beta_{1} \ln \left(1-(1+T)^{-\alpha_{1}}\right)}{R} \\ +\frac{\delta_{1}\left(F_{1}-F_{2}\right) \beta_{1} \ln \left(1-(1+T)^{-\alpha_{1}}\right)}{R^{2}}-\beta_{1} \ln \left(1-(1+T)^{-\alpha_{1}}\right)\end{array}\right)$,

$Q_{125}=Q_{152}=Q_{251}=\frac{(m-s) F_{1} F_{2} \beta_{1} \varphi_{1} \ln \left(1-(1+T)^{-\alpha_{2}}\right)}{R^{2}\left(1-(1+T)^{-\alpha_{1}}\right)}\left(\frac{2\left(1-\delta_{1}\right) \delta_{1}\left(F_{2}-F_{1}\right)}{R}+2 \delta_{1}-1\right)$, 


$$
\begin{aligned}
& Q_{133}=Q_{331}=\frac{(m-s) F_{2} \beta_{2} \varphi_{2}}{R\left(1-(1+T)^{-\alpha_{1}}\right)}\left(-\ln (1+T)-\frac{\left(1-\delta_{1}\right) \ln (1+T)\left(F_{2}-F_{1}\right)}{R}+\frac{\left(1-\delta_{1}\right)\left(\beta_{2}-1\right) \varphi_{2}\left(F_{2}-F_{1}\right)}{R\left(1-(1+T)^{-\alpha_{1}}\right)}+\frac{\left(\beta_{2}-1\right) \varphi_{2}}{\left(1-(1+T)^{-\alpha_{1}}\right)}\right. \\
& \left.+\frac{2\left(1-\delta_{1}\right)^{2} F_{2} \beta_{2} \varphi_{2}\left(F_{2}-F_{1}\right)}{R^{2}\left(1-(1+T)^{-\alpha_{1}}\right)}+\frac{2\left(1-\delta_{1}\right) \beta_{2} \varphi_{2} F_{2}}{R\left(1-(1+T)^{-\alpha_{1}}\right)}\right) \\
& Q_{134}=Q_{341}=Q_{143}=\frac{(m-s) F_{2} F_{1} \beta_{2} \varphi_{2} \ln \left(1-(1+T)^{-\alpha_{1}}\right)}{R^{2}\left(1-(1+T)^{-\alpha_{1}}\right)}\left(\frac{2 \delta_{1}\left(1-\delta_{1}\right)\left(F_{2}-F_{1}\right)}{R}+2 \delta_{1}-1\right), \\
& Q_{135}=Q_{351}=Q_{153}=\frac{(m-s) F_{2} \varphi_{2}}{R\left(1-(1+T)^{-\alpha_{1}}\right)}\left(\begin{array}{c}
1+\beta_{2} \ln \left(1-(1+T)^{-\alpha_{1}}\right)+\frac{\left(1-\delta_{1}\right)\left(F_{2}-F_{1}\right)}{R}+\frac{2\left(1-\delta_{1}\right)^{2} \beta_{2} F_{2}\left(F_{2}-F_{1}\right) \ln \left(1-(1+T)^{-\alpha_{1}}\right)}{R^{2}} \\
+\frac{2 \beta_{2}\left(1-\delta_{1}\right) n\left(1-(1+T)^{-\alpha_{2}}\right) F_{2}}{R}+\frac{\left(1-\delta_{1}\right) \beta_{2}\left(F_{2}-F_{1}\right) \ln \left(1-(1+T)^{-\alpha_{1}}\right)}{R}
\end{array}\right), \\
& Q_{144}=Q_{441}=Q_{414}=\frac{(m-s) F_{2} n\left(1-(1+T)^{-\alpha_{1}}\right) n\left(1-(1+T)^{-\alpha_{2}}\right)}{R^{2}}\left(\frac{2 \delta_{1}^{2} F_{1}\left(F_{2}-F_{1}\right)}{R}-2 \delta_{1} F_{1}+\delta_{1}\left(F_{2}-F_{1}\right)-1\right), \\
& Q_{145}=Q_{451}=\frac{(m-s) F_{2} F_{1} \ln \left(1-(1+T)^{-\alpha_{1}}\right) \ln \left(1-(1+T)^{-\alpha_{2}}\right)}{R^{2}}\left(\frac{2 \delta_{1}\left(1-\delta_{1}\right)\left(F_{2}-F_{1}\right)}{R}+2 \delta_{1}-1\right), \\
& Q_{155}=Q_{551}=\frac{(m-s) F_{2} \ln \left(1-(1+T)^{-\alpha_{2}}\right)^{2}}{R^{2}}\left(1+\frac{2\left(1-\delta_{1}\right) F_{2}}{R}+\frac{2\left(1-\delta_{1}\right) F_{2}}{R^{2}}+\frac{2\left(1-\delta_{1}\right)^{2} F_{2}\left(F_{2}-F_{1}\right)}{R}+\frac{\left(1-\delta_{1}\right)\left(F_{2}-F_{1}\right)}{R}\right) \\
& Q_{222}=-\left(\beta_{1}-1\right)\left(3 \psi_{1111}+2 \psi_{1}^{3}+\psi_{111}\right)+\frac{2 s_{1}}{\alpha_{1}^{3}}+\frac{(m-s) \beta_{1} \delta_{1} \varphi_{1} F_{1}}{\left(1-(1+T)^{-\alpha_{1}}\right) R}\left(-\ln (1+T)^{2}\right)-\frac{2 F_{1}^{2} \beta_{1}^{2} \delta_{1}^{2} \varphi_{1}^{2}}{\left(1-(1+T)^{-\alpha_{1}}\right)^{2} R^{2}}-\frac{F_{1} \beta_{1}\left(2 \beta_{1}-2\right) \delta_{1} \varphi_{1}^{2}}{\left(1-(1+T)^{-\alpha_{1}}\right)^{2} R} \\
& -\frac{2 F_{1}^{2} \beta_{1}^{2} \delta_{1}^{2} \varphi_{1}^{2}}{\left(1-(1+T)^{-\alpha_{1}}\right)^{2} R^{2}}-\frac{F_{1} \beta_{1}\left(2 \beta_{1}-2\right) \delta_{1} \varphi_{1}^{2}}{\left(1-(1+T)^{-\alpha_{1}}\right)^{2} R} \\
& Q_{223}=Q_{332}=\frac{(m-s)\left(1-\delta_{1}\right) \delta_{1} F_{1} F_{2} \beta_{1} \beta_{2} \varphi_{2} \varphi_{1}}{\left(1-(1+T)^{-\alpha_{1}}\right)\left(1-(1+T)^{-\alpha_{2}}\right) R^{2}}\left(\ln (1+T)-\frac{2 \delta_{1} \beta_{1} \varphi_{1} F_{1}}{R\left(1-(1+T)^{-\alpha_{1}}\right)}-\frac{\left(\beta_{1}-1\right) \varphi_{1}}{\left(1-(1+T)^{-\alpha_{1}}\right)}\right) \text {, } \\
& Q_{224}=Q_{242}=\frac{(m-s)\left(1-\delta_{1}\right) \delta_{1} F_{1} F_{2} \beta_{1} \varphi_{1} \ln \left(1-(1+T)^{-\alpha_{2}}\right)}{\left(1-(1+T)^{-\alpha_{1}}\right) R^{2}}\left(\ln (1+T)-\frac{2 \delta_{1} \beta_{1} \varphi_{1} F_{1}}{R\left(1-(1+T)^{-\alpha_{1}}\right)}-\frac{\left(\beta_{1}-1\right) \varphi_{1}}{\left(1-(1+T)^{-\alpha_{1}}\right)}\right) \\
& Q_{225}=Q_{252}=\frac{(m-s)\left(1-\delta_{1}\right) \delta_{1} F_{1} F_{2} \beta_{1} \varphi_{1} \ln \left(1-(1+T)^{-\alpha_{2}}\right)}{\left(1-(1+T)^{-\alpha_{1}}\right) R^{2}}\left(\ln (1+T)-\frac{2 \delta_{1} \beta_{1} \varphi_{1} F_{1}}{R\left(1-(1+T)^{-\alpha_{1}}\right)}-\frac{\left(\beta_{1}-1\right) \varphi_{1}}{\left(1-(1+T)^{-\alpha_{1}}\right)}\right) \\
& Q_{234}=Q_{243}=Q_{342}=-\frac{(m-s)\left(1-\delta_{1}\right) \delta_{1} F_{1} F_{2} \beta_{2} \varphi_{1} \varphi_{2}}{\left(1-(1+T)^{-\alpha_{2}}\right)\left(1-(1+T)^{-\alpha_{1}}\right) R^{2}}\left(1+\beta_{1} \ln \left(1-(1+T)^{-\alpha_{1}}\right)+\frac{2 \delta_{1} \beta_{1} \ln \left(1-(1+T)^{-\alpha_{1}}\right) F_{1}}{R}\right) \\
& Q_{235}=Q_{253}=Q_{352}=-\frac{(m-s)\left(1-\delta_{1}\right) \delta_{1} F_{1} F_{2} \beta_{1} \varphi_{1} \varphi_{2}}{\left(1-(1+T)^{-\alpha_{2}}\right)\left(1-(1+T)^{-\alpha_{1}}\right) R^{2}}\left(1+\beta_{2} \ln \left(1-(1+T)^{-\alpha_{2}}\right)+\frac{2\left(1-\delta_{1}\right) \beta_{2} \ln \left(1-(1+T)^{-\alpha_{2}}\right) F_{2}}{R}\right)
\end{aligned}
$$




$$
\begin{aligned}
& Q_{332}=Q_{233}=Q_{323}=-\frac{(n-r)\left(1-\delta_{1}\right) \delta_{1} F_{1} F_{2} \beta_{1} \beta_{2} \varphi_{1} \varphi_{2}}{\left(1-(1+T)^{-\alpha_{2}}\right)\left(1-(1+T)^{-\alpha_{1}}\right) R^{2}}\left(\ln (1+T)-\frac{2\left(1-\delta_{1}\right) \beta_{2} \varphi_{2} F_{2}}{R\left(1-(1+T)^{-\alpha_{2}}\right)}-\frac{\left(1-\beta_{2}\right) \varphi_{2}}{\left(1-(1+T)^{-\alpha_{2}}\right)}\right), \\
& Q_{244}=Q_{442}=Q_{424}=-\frac{(m-s) \delta_{1} F_{1} \varphi_{1} \ln \left(1-(1+T)^{-\alpha_{2}}\right)}{\left(1-(1+T)^{-\alpha_{1}}\right) R}\left(2+\beta_{2} \ln \left(1-(1+T)^{-\alpha_{2}}\right)+\frac{2 \delta_{1} \beta_{1} F_{1}}{R}+\frac{2 \delta_{1}^{2} \beta_{1} \ln \left(1-(1+T)^{-\alpha_{1}}\right) F_{1}^{2}}{R^{2}}\right. \\
& \left.+\frac{3 \delta_{1} \beta_{1} \ln \left(1-(1+T)^{-\alpha_{1}}\right) F_{1}}{R}\right) \\
& Q_{245}=Q_{254}=Q_{452}=-\frac{(m-s)\left(1-\delta_{1}\right) \delta_{1} F_{1} \varphi_{1} \ln \left(1-(1+T)^{-\alpha_{1}}\right)}{\left(1-(1+T)^{-\alpha_{1}}\right) R^{2}}\left(1+\beta_{1} \ln \left(1-(1+T)^{-\alpha_{2}}\right)+\frac{2 \delta_{1} \beta_{1} \ln \left(1-(1+T)^{-\alpha_{1}}\right) F_{1}}{R}\right), \\
& Q_{255}=Q_{552}=Q_{525}=-\frac{(m-s)\left(1-\delta_{1}\right) \delta_{1} F_{1} F_{2} \varphi_{1} \beta_{1} \varphi_{1}\left(\ln \left(1-(1+T)^{-\alpha_{2}}\right)\right)^{2}}{\left(1-(1+T)^{-\alpha_{1}}\right) R^{2}}\left(1+\frac{2\left(1-\delta_{1}\right) F_{2}}{R}\right), \\
& Q_{222}=-\left(\beta_{2}-1\right)\left(3 \psi_{2222}+2 \psi_{2}^{3}+\psi_{222}\right)+\frac{2 s_{2}}{\alpha_{2}^{3}}+\frac{(m-s) \beta_{2}\left(1-\delta_{1}\right) \varphi_{2} F_{2}}{\left(1-(1+T)^{-\alpha_{2}}\right) R} \\
& \left(-\ln (1+T)^{2}-\frac{2 F_{1}^{2} \beta_{1}^{2} \delta_{1}^{2} \varphi_{1}^{2}}{\left(1-(1+T)^{-\alpha_{1}}\right)^{2} R^{2}}-\frac{F_{1} \beta_{1}\left(2 \beta_{1}-2\right) \delta_{1} \varphi_{1}^{2}}{\left(1-(1+T)^{-\alpha_{1}}\right)^{2} R}-\frac{2 F_{1}^{2} \beta_{1}^{2} \delta_{1}^{2} \varphi_{1}^{2}}{\left(1-(1+T)^{-\alpha_{1}}\right)^{2} R^{2}}-\frac{F_{1} \beta_{1}\left(2 \beta_{1}-2\right) \delta_{1} \varphi_{1}^{2}}{\left(1-(1+T)^{-\alpha_{1}}\right)^{2} R}\right), \\
& Q_{334}=Q_{343}=\frac{(m-s)\left(1-\delta_{1}\right) \delta_{1} F_{1} F_{2} \beta_{2} \varphi_{2} \ln \left(1-(1+T)^{-\alpha_{1}}\right)}{\left(1-(1+T)^{-\alpha_{2}}\right) R^{2}}\left(\ln (1+T)-\frac{2\left(1-\delta_{1}\right) \beta_{2} \varphi_{2} F_{2}}{R\left(1-(1+T)^{-\alpha_{2}}\right)}-\frac{\left(\beta_{2}-1\right) \varphi_{2}}{\left(1-(1+T)^{-\alpha_{2}}\right)}\right), \\
& Q_{344}=Q_{443}=Q_{434}=-\frac{(m-s)\left(1-\delta_{1}\right) \delta_{1} F_{1} F_{2} \beta_{2} \varphi_{2} \ln \left(1-(1+T)^{-\alpha_{1}}\right)^{2}}{\left(1-(1+T)^{-\alpha_{2}}\right) R^{2}}\left(\frac{2 \delta_{1} F_{1}}{R}+1\right) \text {, } \\
& Q_{345}=Q_{354}=Q_{453}=-\frac{(m-s)\left(1-\delta_{1}\right) \delta_{1} F_{2} \varphi_{2} \ln \left(1-(1+T)^{-\alpha_{1}}\right)}{\left(1-(1+T)^{-\alpha_{2}}\right) R^{2}}\left(1+\beta_{2} \ln \left(1-(1+T)^{-\alpha_{2}}\right)\right. \\
& \left.+\frac{2\left(1-\delta_{1}\right) \beta_{2} \ln \left(1-(1+T)^{-\alpha_{2}}\right) F_{2}}{R}\right) \\
& Q_{355}=Q_{553}=Q_{535}=-\frac{(m-s)\left(1-\delta_{1}\right) F_{2} \varphi_{2} \ln \left(1-(1+T)^{-\alpha_{2}}\right)}{\left(1-(1+T)^{-\alpha_{2}}\right) R}\left(2+\beta_{2} \ln \left(1-(1+T)^{-\alpha_{2}}\right)+\frac{2\left(1-\delta_{1}\right) F_{2}}{R}\right. \\
& \left.+\frac{2\left(1-\delta_{1}\right) \beta_{2} \ln \left(1-(1+T)^{-\alpha_{2}}\right) F_{2}^{3}}{R^{2}}+\frac{3\left(1-\delta_{1}\right) \beta_{2} \ln \left(1-(1+T)^{-\alpha_{2}}\right) F_{2}}{R}\right), \\
& Q_{444}=\frac{2 s_{1}}{\beta_{1}^{3}}-\frac{(m-s) \delta_{1} F_{1} \ln \left(1-(1+T)^{-\alpha_{1}}\right)^{2}}{R}\left(\ln \left(1-(1+T)^{-\alpha_{1}}\right)+\frac{3 \delta_{1} F_{1} \ln \left(1-(1+T)^{-\alpha_{1}}\right)}{R}+\frac{2 \delta_{1}^{2} F_{1}^{2} \ln \left(1-(1+T)^{-\alpha_{1}}\right)}{R^{2}}\right) \text {, } \\
& Q_{445}=Q_{454}=\frac{(m-s) \delta_{1}\left(1-\delta_{1}\right) F_{1} F_{2} \ln \left(1-(1+T)^{-\alpha_{2}}\right) \ln \left(1-(1+T)^{-\alpha_{1}}\right)^{2}}{R^{2}}\left(\frac{2 \delta_{1} F_{1}}{R}+1\right), \\
& Q_{455}=Q_{554}=Q_{545}=\frac{(m-s) \delta_{1}\left(1-\delta_{1}\right) F_{1} F_{2} \ln \left(1-(1+T)^{-\alpha_{2}}\right)^{2} \ln \left(1-(1+T)^{-\alpha_{1}}\right)}{R^{2}}\left(\frac{2\left(1-\delta_{1}\right) F_{2}}{R}+1\right) \text {, } \\
& Q_{555}=\frac{2 s_{2}}{\beta_{2}^{3}}-\frac{(m-s)\left(1-\delta_{1}\right) F_{2} \ln \left(1-(1+T)^{-\alpha_{2}}\right)^{2}}{R}\left(\ln \left(1-(1+T)^{-\alpha_{2}}\right)+\frac{3\left(1-\delta_{1}\right) F_{2}}{R}+\frac{2\left(1-\delta_{1}\right)^{2} F_{1}^{2}}{R^{2}}\right) \text {, }
\end{aligned}
$$




$$
\begin{aligned}
D_{\mathrm{LLF}} & =\frac{\partial U(\Theta)}{\partial \Theta}=U_{11}, U_{22}, U_{33}, U_{44}, U_{55}, \\
\text { if } \Theta & =\delta_{1} \text { than, } U_{11}=\frac{\partial}{\partial \delta_{1}}\left(e^{-\eta \delta_{1}}\right)=\delta_{1}^{2} e^{-\eta \delta_{1}}, \\
\Theta & =\alpha_{1}, U_{11}=\frac{\partial}{\partial \alpha_{1}}\left(e^{-\eta \alpha_{1}}\right)=\alpha_{1}^{2} e^{-\eta \alpha_{1}}, \\
\Theta & =\alpha_{2}, U_{22}=\frac{\partial}{\partial \alpha_{2}}\left(e^{-\eta \alpha_{2}}\right)=\alpha_{2}^{2} e^{-\eta \alpha_{2}}, \\
\Theta & =\beta_{1}, U_{33}=\frac{\partial}{\partial \beta_{1}}\left(e^{-\eta \beta_{1}}\right)=\beta_{1}^{2} e^{-\eta \beta_{1}}, \\
\Theta & =\beta_{2}, U_{55}=\frac{\partial}{\partial \beta_{2}}\left(e^{-\eta \beta_{2}}\right)=\beta_{2}^{2} e^{-\eta \beta_{2}} .
\end{aligned}
$$

\section{Data Availability}

No data were used to support this study.

\section{Conflicts of Interest}

The authors declare that they have no conflicts of interest.

\section{References}

[1] S. Ali, M. Aslam, D. Kundu, and S. M. A. Kazmi, "Bayesian estimation of the mixture of generalized exponential distribution: a versatile lifetime model in industrial processes," Journal of the Chinese Institute of Industrial Engineers, vol. 29, no. 4, pp. 246-269, 2012.

[2] C. G. Bhattacharya, "A simple method of resolution of a distribution into Gaussian components," Biometrics, vol. 23, no. 1, pp. 115-135, 1967.

[3] D. R. Harris, "The development of socio-legal studies in the United Kingdom,” Legal Studies, vol. 3, no. 3, pp. 315-333, 1983.

[4] K. Jedidi, H. S. Jagpal, and W. S. DeSarbo, "Finite-mixture structural equation models for response-based segmentation and unobserved heterogeneity," Marketing Science, vol. 16, no. 1, pp. 39-59, 1997.

[5] K. S. Sultan, M. A. Ismail, and A. S. Al-Moisheer, "Mixture of two inverse Weibull distributions: properties and estimation," Computational Statistics and Data, vol. 51, no. 1, pp. 53775387, 2007.

[6] B. S. Everitt, "Mixture distributions-I," in Encyclopedia of Statistical SciencesJohn Wiley and Sons, Inc., New York, NY, USA, 2004.

[7] S. Ali, "Mixture of the inverse Rayleigh distribution: properties and estimation in Bayesian framework," Applied Mathematical Modelling, vol. 39, no. 2, pp. 515-530, 2014.

[8] N. Feroze and M. Aslam, "Bayesian analysis of doubly censored lifetime data using two component mixture of Weibull distribution," Journal of the National Science Foundation of Sri Lanka, vol. 42, no. 4, pp. 325-334, 2014.

[9] H. Zhang and Y. Huang, "Finite mixture models and their applications: a review," Austin Biometrics and Biostatistics, vol. 2, no. 1, pp. 1-6, 2015.

[10] M. D. Fundi, E. G. Njenga, and K. G. Keitany, "Estimation of parameters of the two-parameter Rayleigh distribution based on progressive type-II censoring using maximum likelihood method via the NR and the EM algorithms," American Journal of Theoretical and Applied Statistics, vol. 6, no. 1, pp. 1-9, 2017.
[11] Y. M. Tripathi, C. Petropoulos, F. Sultana, and M. K. Rastogi, "Estimating a linear parametric function of a doubly censored exponential distribution," Statistics, vol. 52, no. 1, pp. 99-114, 2018.

[12] F. Noor, A. Sajid, S. B. H. Shah, M. Zaman, M. Gheisari, and V. Mariappan, "Bayesian estimation and prediction for BurrRayleigh mixture model using censored data," International Journal of Communication Systems, vol. 32, no. 15, p. 4094, 2019.

[13] N. Feroze and M. Aslam, "Approximate Bayesian analysis of doubly censored samples from mixture of two Weibull distributions," Communications in Statistics - Theory and Methods, vol. 48, no. 11, pp. 2862-2878, 2019.

[14] A. Aljuaid, "Estimating the parameters of an exponentiated inverted Weibull distribution under type-II censoring," Applied Mathematical Sciences, vol. 7, no. 35, pp. 1721-1736, 2013.

[15] F. Noor and M. Aslam, "Bayesian inference of the inverse Weibull mixture distribution using type-I censoring," Journal of Applied Statistics, vol. 40, no. 5, pp. 1076-1089, 2013.

[16] R. I. Abd ELs-Kader, G. R. AL-Dayian, and S. A. AL-Gendy, "Inverted Pareto type I distribution: properties and estimation," Journal of Faculty of Commerce AL-Azhar University, vol. 21, pp. 19-40, 2003.

[17] A. M. Basheer, "Alpha power inverse Weibull distribution with reliability application," Journal of Taibah University for Science, vol. 13, no. 1, pp. 423-432, 2019.

[18] A. S. Hassan and A. N. Zaky, "Estimation of entropy for inverse Weibull distribution under multiple censored data," Journal of Taibah University for Science, vol. 13, no. 1, pp. 331-337, 2019.

[19] P. Kumaraswamy, "A generalized probability density function for double-bounded random processes," Journal of Hydrology, vol. 46, no. 1-2, pp. 79-88, 1980.

[20] A. M. AL-Fattah, A. A. EL-Helbawy, and G. R. AL-Dayian, "Inverted Kumaraswamy distribution: properties and estimation," Pakistan Journal of Statistics (PJS), vol. 33, no. 1, pp. 37-61, 2017.

[21] W. Mendenhall and R. J. Hader, "Estimation of parameters of mixed exponentially distributed failure time distributions from censored life test data," Biometrika, vol. 45, no. 3-4, pp. 504-520, 1958.

[22] D. V. Lindley, "Approximate bayesian methods," Trabajos de Estadistica $Y$ de Investigacion Operativa, vol. 31, no. 1, pp. 223-245, 1980. 
[23] Z. F. Jaheen, "Bayesian estimations and predictions based on single Burr type XII models and their finite mixture," Ph. D. thesis, Egypt University of Assiut, Assiut, Egypt, 1993.

[24] K. E. Ahmad, H. M. Moustafa, and A. M. Abd-Elrahman, "Approximate Bayes estimation for mixtures of two Weibull distributions under type-2 censoring," Journal of Statistical Computation and Simulation, vol. 58, no. 3, pp. 269-285, 1997.

[25] K. S. Sultan and A. S. Al-Moisheer, "Approximate Bayes estimation of the parameters and reliability function of a mixture of two inverse Weibull distributions under type-2 censoring," Journal of Statistical Computation and Simulation, vol. 83, no. 10, pp. 1900-1914, 2013. 